\title{
Bifurcations and Parametric Representations of Peakon, Solitary Wave and Smooth Periodic Wave Solutions for a Two-Component Degasperis-Procesi Equation
}

\author{
Bin He, ${ }^{1}$ Qing Meng, ${ }^{2}$ and Jinhua Zhang ${ }^{1}$ \\ ${ }^{1}$ College of Mathematics, Honghe University, Mengzi, Yunnan 661100, China \\ ${ }^{2}$ Department of Physics, Honghe University, Mengzi, Yunnan 661100, China \\ Correspondence should be addressed to Bin He; hebinmtc@163.com
}

Received 27 May 2013; Revised 26 July 2013; Accepted 26 July 2013

Academic Editor: Jingxin Zhang

Copyright (C) 2013 Bin He et al. This is an open access article distributed under the Creative Commons Attribution License, which permits unrestricted use, distribution, and reproduction in any medium, provided the original work is properly cited.

By using the bifurcation method of dynamical systems and the method of phase portraits analysis, we consider a two-component Degasperis-Procesi equation: $m_{t}=-3 m u_{x}-m_{x} u+k \rho \rho_{x}, \rho_{t}=-\rho_{x} u+2 \rho u_{x}$, the existence of the peakon, solitary wave and smooth periodic wave is proved, and exact parametric representations of above travelling wave solutions are obtained in different parameter regions.

\section{Introduction}

Based on the deformation of bi-Hamiltonian structure of the hydrodynamic type, Chen et al. [1] obtained a two-component Camassa-Holm equation:

$$
\begin{gathered}
m_{t}=-2 m u_{x}-m_{x} u+e \rho \rho_{x}, \\
\rho_{t}=-\rho_{x} u-\rho u_{x},
\end{gathered}
$$

where $m=u-u_{x x}$ and $e= \pm 1$. This system is integrable in the sense that it has Lax pair and it is a generalization form of the Camassa-Holm equation [2]. A good fact is that it has the peakon and multikink solutions [3].

A two-component generalization of the DegasperisProcesi equation $[4,5]$ is read as follows:

$$
\begin{gathered}
m_{t}=-3 m u_{x}-m_{x} u+k \rho \rho_{x}, \\
\rho_{t}=-\rho_{x} u+2 \rho u_{x},
\end{gathered}
$$

where $m=u-u_{x x}, k$ is a real parameter. When $\rho=0$, (2) is reduced to the Degasperis-Procesi equation [6]. Jin and Guo [4] analyze some aspects of blowup mechanism, traveling wave solutions and the persistence properties for (2). The selfsimilar solutions of (2) have been obtained by yuen [5].
In the present paper, we will investigate the bifurcation set of (2) using the bifurcation theory and the method of phase portraits analysis [7-9] and obtain some exact peakon, solitary wave and smooth periodic wave solutions.

Let $\xi=x-c t$, where $c \neq 0$ is the wave speed. By using the travelling wave transformation

$$
\begin{gathered}
u(x, t)=\phi(\xi)+\frac{4}{3} c, \\
\rho(x, t)=\rho(\xi),
\end{gathered}
$$

we reduce (2) to the following ordinary differential equations:

$$
\begin{gathered}
\left(\phi+\frac{1}{3} c\right) \phi^{\prime \prime \prime}+3 \phi^{\prime} \phi^{\prime \prime}-4 \phi \phi^{\prime}-\frac{13}{3} c \phi^{\prime}+k \rho \rho^{\prime}=0, \\
2 \rho \phi^{\prime}-\left(\phi+\frac{1}{3} c\right) \rho^{\prime}=0,
\end{gathered}
$$

where " $I$ " is the derivative with respect to $\xi$.

Suppose that

$$
\rho=\left(\phi+\frac{4}{3} c\right)^{2}+a_{1}\left(\phi+\frac{4}{3} c\right)+a_{0}
$$

where $a_{1}, a_{0}$ are constants to be determined later. 
Substituting (5) into the second equation of (4), we have

$$
\left(2 c+a_{1}\right) \phi \phi^{\prime}+\left(\frac{8}{3} c^{2}+\frac{7}{3} a_{1} c+2 a_{0}\right) \phi^{\prime}=0 .
$$

Integrating (6) once with respect to $\xi$ and setting the integral constant to zero yield

$$
\frac{1}{2}\left(2 c+a_{1}\right) \phi^{2}+\left(\frac{8}{3} c^{2}+\frac{7}{3} a_{1} c+2 a_{0}\right) \phi=0 .
$$

From (7), we obtain

$$
2 c+a_{1}=0, \quad \frac{8}{3} c^{2}+\frac{7}{3} a_{1} c+2 a_{0}=0 .
$$

Solving (8), we find the following set of solutions:

$$
a_{1}=-2 c, \quad a_{0}=c^{2} .
$$

Substituting (9) into (5), we have

$$
\rho=\left(\phi+\frac{1}{3} c\right)^{2} .
$$

Substituting (10) into the first equation of (4), integrating once with respect to $\xi$, and setting the integral constant to $(1 / 162) k c^{4}-(32 / 9) c^{2}$ yield

$$
\begin{aligned}
(\phi & \left.+\frac{1}{3} c\right) \phi^{\prime \prime}-\left(\phi+\frac{4}{3} c\right) \\
& \times\left(-\frac{1}{2} k \phi^{3}+\left(2-\frac{1}{3} k c^{2}\right) \phi+\frac{10}{27} k c^{3}+\frac{5}{3} c\right)+\left(\phi^{\prime}\right)^{2}=0 .
\end{aligned}
$$

Letting $y=d \phi / d \xi$, we get the following planar system:

$$
\begin{gathered}
\frac{d \phi}{d \xi}=y, \\
\frac{d y}{d \xi}=\left(\left(\phi+\frac{4}{3} c\right)\right. \\
\left.\times\left(-\frac{1}{2} k \phi^{3}+\left(2-\frac{1}{3} k c^{2}\right) \phi+\frac{10}{27} k c^{3}+\frac{5}{3} c\right)-y^{2}\right) \\
\times\left(\phi+\frac{1}{3} c\right)^{-1} .
\end{gathered}
$$

System (12) is a two-parameter planar dynamical system depending on the parameter set $(c, k)$. Since the phase orbits defined by the vector field of system (12) determine all travelling wave solutions of (11), we should investigate the bifurcations of phase portraits of system (12) in $(\phi, y)$-phase plane as the parameters $c, k$ are changed.

Clearly, on such straight line $\phi=-(1 / 3) c$ in the phase plane $(\phi, y)$, system (12) is discontinuous. Such system is called a singular travelling wave system by one of authors in [10].
Definition 1 (see $[7,10]$ ). Suppose that $\phi(\xi)$ is a solution of system (12) for $\xi \in(-\infty, \infty)$. (i) $\phi(\xi)$ is called peakon solution if $\phi(\xi)$ is smooth locally on either side of $\xi_{0}$ and $\lim _{\xi \rightarrow \xi_{0}-0} \phi(\xi)=\lim _{\xi \rightarrow \xi_{0}+0} \phi(\xi)=a$ and $a \neq 0, a \neq \pm \infty$. (ii) $\phi(\xi)$ is called a solitary wave solution if $\lim _{\xi \rightarrow+\infty} \phi(\xi)=$ $\lim _{\xi \rightarrow-\infty} \phi(\xi)$. Usually, a peakon solution of (2) corresponds to two heteroclinic orbits of system (12) and a solitary wave solution of (2) corresponds to a homoclinic orbit of system (12). Similarly, a periodic orbit of system (12) corresponds to a smooth periodic wave solution of (2).

Thus, to investigate peakons, solitary waves and smooth periodic waves of (2), we should find all heteroclinic, homoclinic, and periodic orbits of system (12) depending on the parameter space of this system.

The rest of this paper is organized as follows. In Section 2, we discuss the bifurcations of phase portraits of system (12), where explicit parametric conditions will be derived. In Section 3, we give some exact parametric representations of peakon, solitary wave and smooth periodic wave solutions of (2). A short conclusion will be given in Section 4.

\section{Bifurcation Sets and Phase Portraits of System (12)}

Using the transformation $d \xi=(\phi+(1 / 3) c) d \tau$, it carries (12) into the Hamiltonian system

$$
\begin{gathered}
\frac{d \phi}{d \tau}=\left(\phi+\frac{1}{3} c\right) y, \\
\frac{d y}{d \tau}=\left(\phi+\frac{4}{3} c\right) \\
\quad \times\left(-\frac{1}{2} k \phi^{3}+\left(2-\frac{1}{3} k c^{2}\right) \phi+\frac{10}{27} k c^{3}+\frac{5}{3} c\right)-y^{2} .
\end{gathered}
$$

Since both systems (12) and (13) have the same first integral

$$
\begin{aligned}
(3 \phi+c)^{2} y^{2} & \\
+\frac{1}{54} \phi( & 81 k \phi^{5}+162 k c \phi^{4}+9\left(15 k c^{2}-52\right) \phi^{3} \\
+ & 60 c\left(k c^{2}-27\right) \phi^{2}-2 c^{2}\left(114 k c^{2}+891\right) \phi \\
- & \left.80 c^{3}\left(2 k c^{2}+9\right)\right)=h,
\end{aligned}
$$

then two systems above have the same topological phase portraits except for the line $\phi=-(1 / 3) c$. Therefore, we can obtain the bifurcation phase portraits of system (12) from that of system (13). We consider the equilibrium points and their properties for system (13) as follows.

When $k=0$, it is easy to see that system (13) has two equilibrium points $(-(4 / 3) c, 0),(-(5 / 6) c, 0)$ on $\phi$-axis and two equilibrium points $(-(1 / 3) c,-c),(-(1 / 3) c, c)$ on the line $\phi=-(1 / 3) c$. 


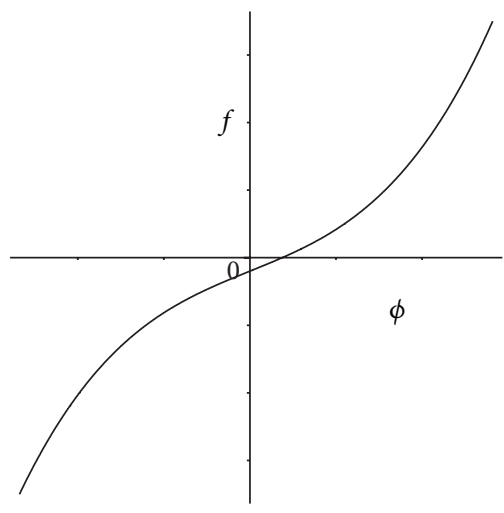

(a)

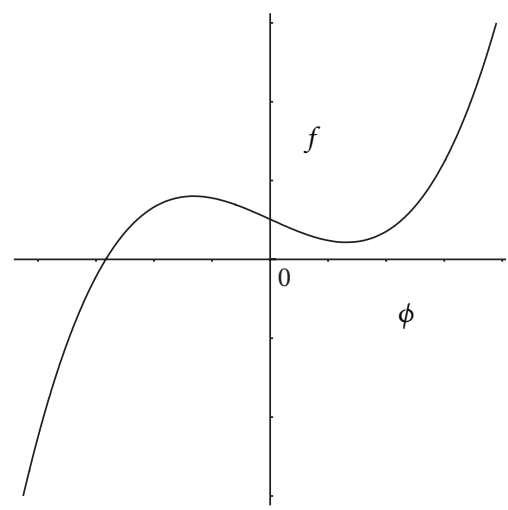

(d)

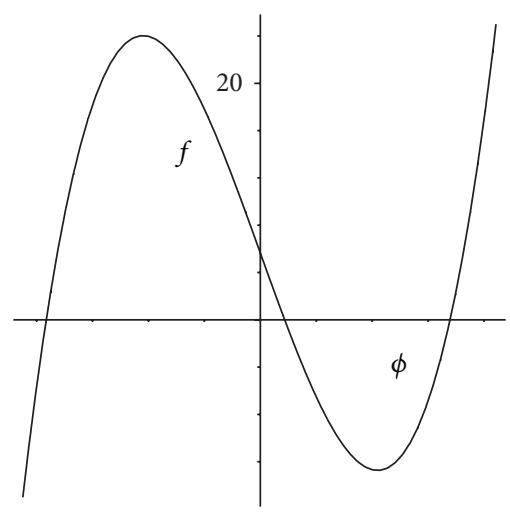

(b)

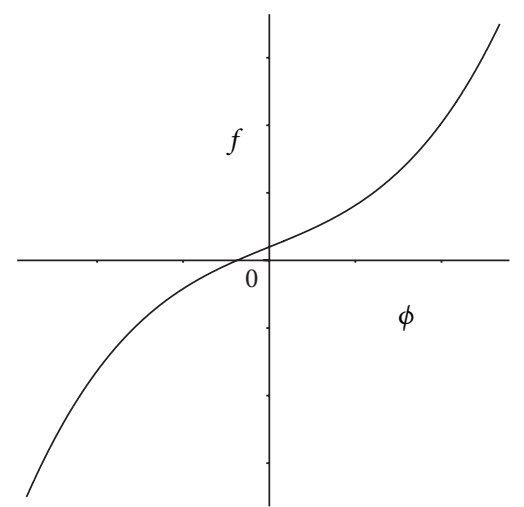

(e)

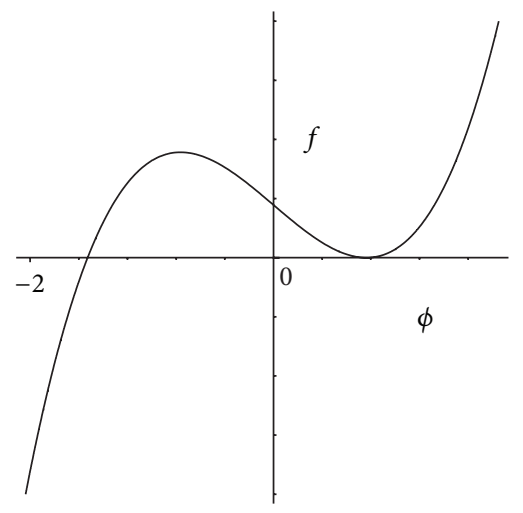

(c)

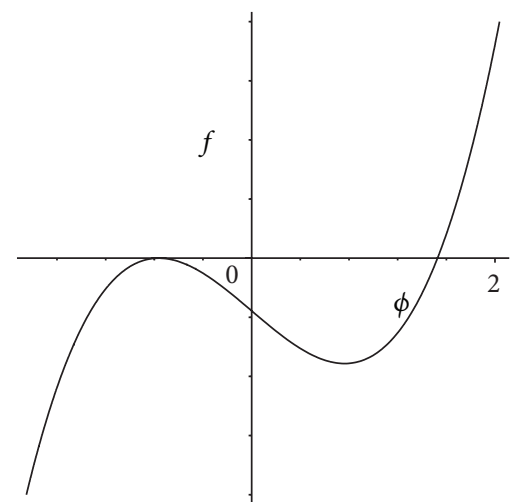

(g)

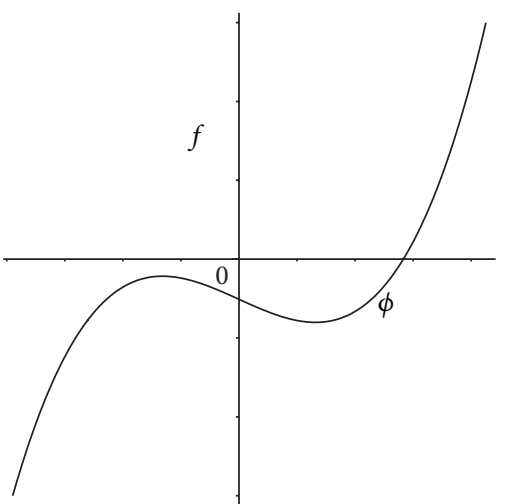

(h)

Figure 1: The graphics of the function $f(\phi)$ when $c \neq 0$. (a) $c<0, k<0$. (b) $c<0,0<k<k_{*}$. (c) $c<0, k=k_{*}$. (d): $c<0, k>k_{*}$. (e) $c>0$, $k<0$. (f) $c>0,0<k<k_{*}$. (g) $c>0, k=k_{*}$. (h) $c>0, k>k_{*}$.

When $k \neq 0$, let

$$
f(\phi)=\phi^{3}-\frac{2}{k}\left(2-\frac{1}{3} k c^{2}\right) \phi-\frac{10}{3 k}\left(\frac{2}{9} k c^{2}+1\right) .
$$

Obviously, $(-(4 / 3) c, 0)$ is an equilibrium point of system (13) and $(\phi, 0)$ is a equilibrium point of system (13) if and only if $f(\phi)=0$ when $\phi \neq-(4 / 3) c$. Denoting that $\Omega=(5725+$ $1575 \sqrt{14})^{1 / 3}, k_{*}=\left(\Omega^{2}-14 \Omega-125\right) / 6 \Omega c^{2}$, we draw the graph of $f=f(\phi)$ for given $c \neq 0$ as Figure 1 .

From Figure 1 and paying attention to that $(-(4 / 3) c, 0)$ is always an equilibrium point of system (13), we have the following. (i) If $k<0$, then system (13) has two equilibrium points on $\phi$-axis.

(ii) If $0<k<k_{*}$, then system (13) has four equilibrium points on $\phi$-axis.

(iii) If $k=k_{*}$, then system (13) has three equilibrium points on $\phi$-axis.

(iv) If $k>k_{*}$, then system (13) has two equilibrium points on $\phi$-axis.

On the other hand, it is also easy to see that if $k>$ $-2 / c^{2}$, then system (13) has two equilibrium points $(-(1 / 3) c$, 
$\left.\pm(\sqrt{2} c / 2) \sqrt{k c^{2}+2}\right)$ on the line $\phi=-(1 / 3) c$ and if $k=-2 / c^{2}$, then system (13) has one equilibrium point $(-(1 / 3) c, 0)$ on the line $\phi=-(1 / 3) c$.

Let $M\left(\phi_{e}, y_{e}\right)$ be the coefficient matrix of the linearized system of the system (13) at an equilibrium point $\left(\phi_{e}, y_{e}\right)$. Then, we have

$$
\begin{aligned}
& M\left(\phi_{e}, y_{e}\right) \\
& =\left(\begin{array}{cc}
y_{e} & \phi_{e}+\frac{1}{3} c \\
-2 k \phi_{e}^{3}-2 k c \phi_{e}^{2}+2\left(2-\frac{1}{3} k c^{2}\right) \phi_{e}+\frac{1}{3} c\left(13-\frac{2}{9} k c^{2}\right) & -2 y_{e}
\end{array}\right),
\end{aligned}
$$

and at this equilibrium point, we have

$$
\begin{aligned}
\operatorname{Trace}\left(M\left(\phi_{e}, y_{e}\right)\right)= & -y_{e}, \\
J\left(\phi_{e}, y_{e}\right)= & \operatorname{det} M\left(\phi_{e}, y_{e}\right) \\
= & -2 y_{e}^{2}+2 k \phi_{e}^{4}+k c \phi_{e}^{3}+4\left(\frac{1}{3} k c^{2}-1\right) \phi_{e}^{2} \\
& +\frac{1}{3} c\left(\frac{8}{9} k c^{2}-17\right) \phi_{e} \\
& +\frac{1}{9} c^{2}\left(\frac{2}{9} k c^{2}-13\right) .
\end{aligned}
$$

By the theory of planar dynamical systems, we know that for an equilibrium point $\left(\phi_{e}, y_{e}\right)$ of a planar integrable system if $J\left(\phi_{e}, y_{e}\right)<0$, then the equilibrium point is a saddle point. If $J\left(\phi_{e}, y_{e}\right)>0$ and $\operatorname{Trace}\left(M\left(\phi_{e}, y_{e}\right)\right)=0$, then it is a center point. If $J\left(\phi_{e}, y_{e}\right)=0$ and the Poincaré index of the equilibrium point is zero, then it is a cusp.

For a fixed $h$, the level curve $H(\phi, y)=h$ defined by (14) determines a set of invariant curves of system (13) which contains different branches of curves. As $h$ is varied, it defines different families of orbits of (13) with different dynamical behaviors.

Using the property of the equilibrium points and bifurcation theory, we obtain the following results.

(i) When $k=0$, there is one bifurcation line $c=0$ which divides the region $(-\infty, \infty)$ into 2 subregions: $A_{1}=$ $\{c \mid c<0\}, A_{2}=\{c \mid c>0\}$.

(ii) When $k \neq 0$, there are five bifurcation curves as follows:

$$
\begin{gathered}
L_{1}: c=0, \quad L_{2}: k=0, \quad L_{3}: k=\frac{\mu}{c^{2}}, \\
L_{4}: k=\frac{1}{2 c^{2}}, \quad L_{5}: k=-\frac{2}{c^{2}},
\end{gathered}
$$

which divide the $(c, k)$-parameter plane into 16 subregions as follows:

$$
\begin{aligned}
& B_{1}=\left\{(c, k) \mid c<0, k<-2 / c^{2}\right\}, \\
& B_{2}=\left\{(c, k) \mid c<0, k=-2 / c^{2}\right\}, \\
& B_{3}=\left\{(c, k) \mid c<0,-2 / c^{2}<k<0\right\}, \\
& B_{4}=\left\{(c, k) \mid c<0,0<k<1 / 2 c^{2}\right\}, \\
& B_{5}=\left\{(c, k) \mid c<0, k=1 / 2 c^{2}\right\}, \\
& B_{6}=\left\{(c, k) \mid c<0,1 / 2 c^{2}<k<\mu / c^{2}\right\} \text {, } \\
& B_{7}=\left\{(c, k) \mid c<0, k=\mu / c^{2}\right\}, \\
& B_{8}=\left\{(c, k) \mid c<0, k>\mu / c^{2}\right\} \text {, } \\
& B_{9}=\left\{(c, k) \mid c>0, k<-2 / c^{2}\right\}, \\
& B_{10}=\left\{(c, k) \mid c>0, k=-2 / c^{2}\right\}, \\
& B_{11}=\left\{(c, k) \mid c>0,-2 / c^{2}<k<0\right\} \text {, } \\
& B_{12}=\left\{(c, k) \mid c>0,0<k<1 / 2 c^{2}\right\} \text {, } \\
& B_{13}=\left\{(c, k) \mid c>0, k=1 / 2 c^{2}\right\}, \\
& B_{14}=\left\{(c, k) \mid c>0,1 / 2 c^{2}<k<\mu / c^{2}\right\} \text {, } \\
& B_{15}=\left\{(c, k) \mid c>0, k=\mu / c^{2}\right\}, \\
& B_{16}=\left\{(c, k) \mid c>0, k>\mu / c^{2}\right\},
\end{aligned}
$$

where $\mu=\left(\Omega^{2}-14 \Omega-125\right) / 6 \Omega, \Omega=(5725+1575 \sqrt{14})^{1 / 3}$.

The bifurcation sets and phase portraits of system (13) are shown in Figures 2 and 3.

\section{Exact Parametric Representations of Peakon, Solitary Wave and Smooth Periodic Wave Solutions of (2)}

In this section, we present all possible exact parametric representations of peakon, solitary wave and smooth periodic wave solutions through some special phase orbits. Next, we always suppose that $h_{1}=\left((16 / 9)-(608 / 243) k c^{2}\right) c^{4}$, $h_{2}=\left(c^{4} / 23328000\right)\left((895537-238014 \sqrt{14}) \Omega^{2}+(970075+\right.$ $382725 \sqrt{14}) \Omega-45865625), \phi_{s}=-(1 / 3) c, \phi_{1}=-(4 / 3) c$, $\phi_{2}=((1 / 1500) c)\left((6 \sqrt{14}-23) \Omega^{2}-5(3 \sqrt{14}+1) \Omega-625\right)$, and $\Omega=(5725+1575 \sqrt{14})^{1 / 3}$.

3.1. Exact Parametric Representations of Peakon Solutions. (i) For the given $h=(16 / 9) c^{4}$ in Figure 2(a), the level curve is shown in Figure 4(a). From Figure 4(a), we see that there are two heteroclinic orbits connecting with saddle points $\left(\phi_{s}, \pm c\right)$ and $\left(\phi_{1}, 0\right)$ of system (13) when $(c, k) \in A_{1}$. Their expressions are

$$
y= \pm\left(\phi-\phi_{1}\right), \quad \phi_{s} \leq \phi<\phi_{1}
$$




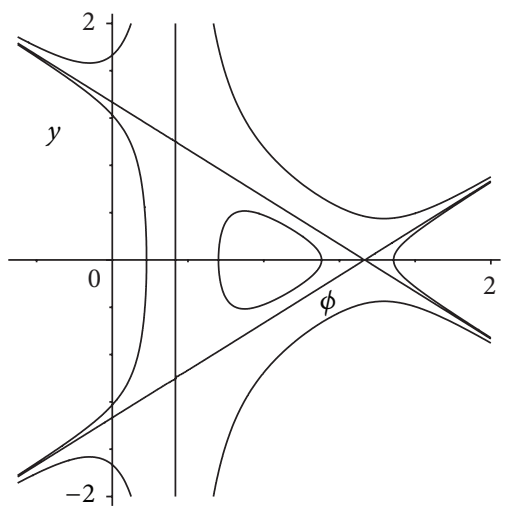

(a)

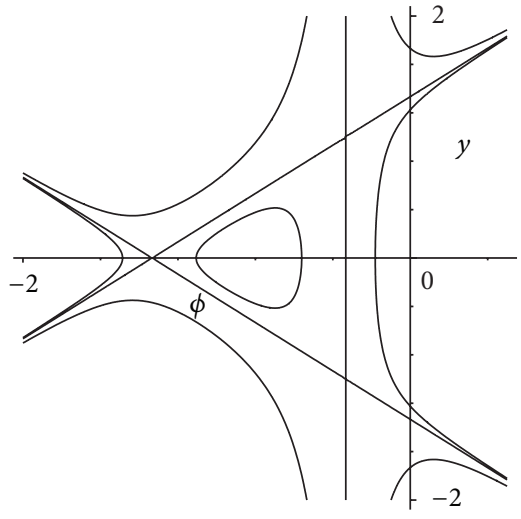

(b)

FIgURE 2: The bifurcation sets and phase portraits of system (13) when $k=0$. (a) $c \in A_{1}$. (b) $c \in A_{2}$.

Substituting (20) into the $d \phi / d \xi=y$ and integrating it along the heteroclinic orbits yield

$$
\int_{\phi_{s}}^{\phi} \frac{d s}{s-\phi_{1}}=-|\xi| .
$$

Completing the above integral and solving the equation for $\phi$, it follows that

$$
\phi(\xi)=c \mathrm{e}^{-|\xi|}-\frac{4}{3} c .
$$

Noting that $u(x, t)=\phi(x-c t)+(4 / 3) c, \rho(x, t)=(\phi(\xi)+$ $(1 / 3) c)^{2}$, and $\xi=x-c t$, we get the parametric representation of peakon solution as follows:

$$
\begin{gathered}
u(x, t)=c \mathrm{e}^{-|x-c t|}, \\
\rho(x, t)=c^{2}\left(\mathrm{e}^{-|x-c t|}-1\right)^{2} .
\end{gathered}
$$

(ii) For the given $h=(16 / 9) c^{4}$ in Figure 2(b), the level curve is shown in Figure 4(b). From Figure 4(b), we see that there are two heteroclinic orbits connecting with saddle points $\left(\phi_{s}, \pm c\right)$ and $\left(\phi_{1}, 0\right)$ of system (13) when $(c, k) \in A_{2}$. Their expressions are

$$
y= \pm\left(\phi-\phi_{1}\right), \quad \phi_{1}<\phi \leq \phi_{s} .
$$

Substituting (24) into the $d \phi / d \xi=y$ and integrating it along the heteroclinic orbits yield

$$
\int_{\phi}^{\phi_{s}} \frac{d s}{s-\phi_{1}}=|\xi|
$$

Completing the above integral, we can get the parametric representation of peakon solution which is the same as (23).

3.2. Exact Parametric Representations of Solitary Wave Solutions. (i) For the given $h=h_{1}$ in Figure 3(d), the level curve is shown in Figure 4(c). From Figure 4(c), we see that there is a homoclinic orbit connecting with a saddle point $\left(\phi_{1}, 0\right)$ of system (13) and passing point $\left(\beta_{2}, 0\right)$, and at the same time, there is a homoclinic orbit connecting with the saddle point $\left(\phi_{1}, 0\right)$ and passing point $\left(\beta_{1}, 0\right)$ when $(c, k) \in B_{4}$, and their expressions are

$$
\begin{gathered}
y= \pm \sqrt{\frac{k}{6} \frac{\left(\phi-\phi_{1}\right) \sqrt{\left(\beta_{1}-\phi\right)\left(\phi-\beta_{2}\right)\left(\phi-\beta_{3}\right)\left(\phi-\beta_{4}\right)}}{\phi-\phi_{s}},}, \\
\beta_{2} \leq \phi<\phi_{1}, \\
y= \pm \sqrt{\frac{k}{6} \frac{\left(\phi-\phi_{1}\right) \sqrt{\left(\beta_{1}-\phi\right)\left(\phi-\beta_{2}\right)\left(\phi-\beta_{3}\right)\left(\phi-\beta_{4}\right)}}{\phi-\phi_{s}},} \\
\phi_{1}<\phi \leq \beta_{1},
\end{gathered}
$$

respectively, where $\beta_{1}, \beta_{2}, \beta_{3}$, and $\beta_{4}\left(\beta_{4}<\beta_{3}<\beta_{2}<\beta_{1}\right)$ are four real roots of

$$
\begin{aligned}
\psi^{4}- & \frac{2}{3} c \psi^{3}+\frac{1}{3 k}\left(5 k c^{2}-18\right) \psi^{2} \\
& -\frac{4 c}{27 k}\left(17 k c^{2}+27\right) \psi+\frac{2 c^{2}}{81 k}\left(38 k c^{2}-27\right)=0,
\end{aligned}
$$

which are obtained by the Cardan formula. For example, $\beta_{1} \approx$ $3.392753049, \beta_{2} \approx 0.8389789971, \beta_{3} \approx 0.09041647394, \beta_{4} \approx$ -4.988815187 when $c=-1, k=0.3 ; \beta_{1} \approx 1.942359082, \beta_{2} \approx$ $0.3828621123, \beta_{3} \approx 0.05356030588, \beta_{4} \approx-2.712114834$ when $c=-0.5, k=1$; and $\beta_{1} \approx 2.712114834, \beta_{2} \approx$ $-0.05356030588, \beta_{3} \approx-0.3828621123, \beta_{4} \approx-1.942359082$ when $c=0.5, k=1$.

Substituting (26) into the $d \phi / d \xi=y$ and integrating it along the homoclinic orbit yield

$$
\begin{aligned}
\int_{\beta_{2}}^{\phi} & \frac{\left(s-\phi_{s}\right) d s}{\left(s-\phi_{1}\right) \sqrt{\left(\beta_{1}-s\right)\left(s-\beta_{2}\right)\left(s-\beta_{3}\right)\left(s-\beta_{4}\right)}} \\
& =-\sqrt{\frac{k}{6}}|\xi| .
\end{aligned}
$$




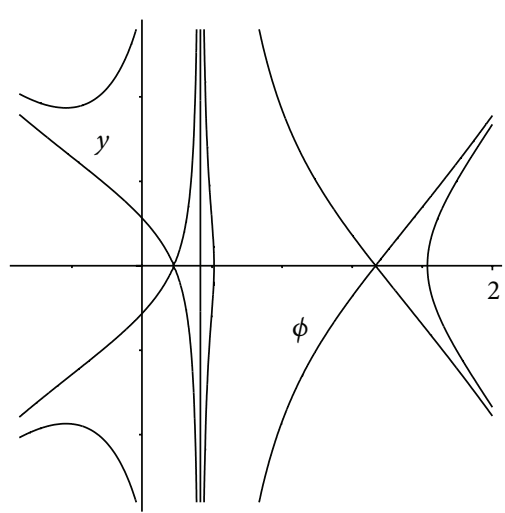

(a)

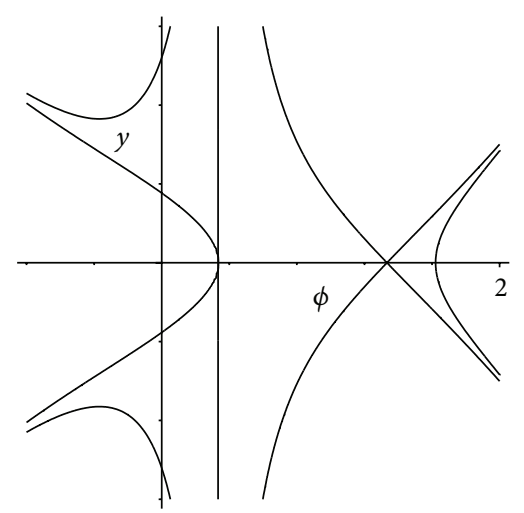

(b)

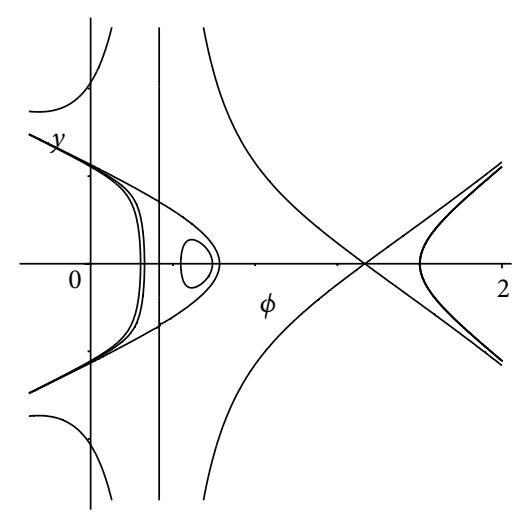

(c)

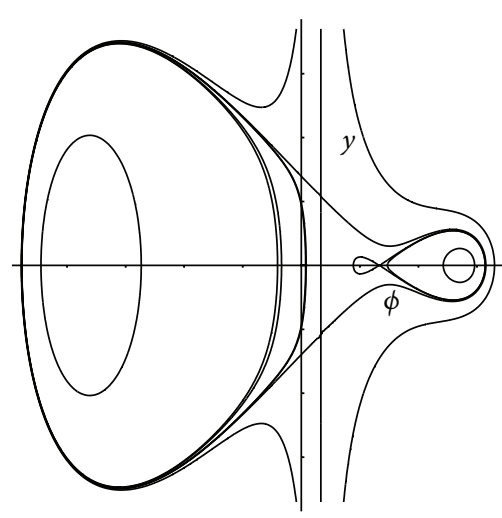

(d)

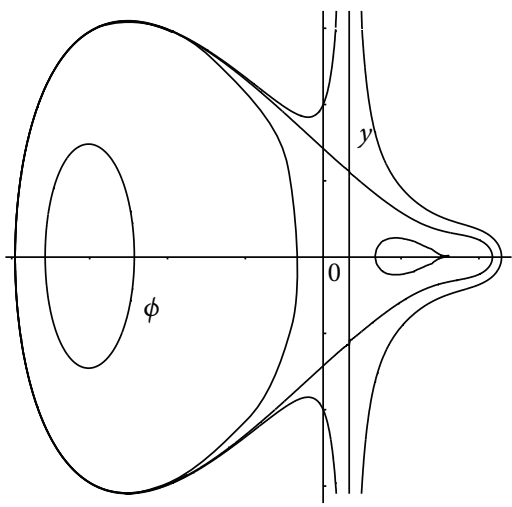

(g)

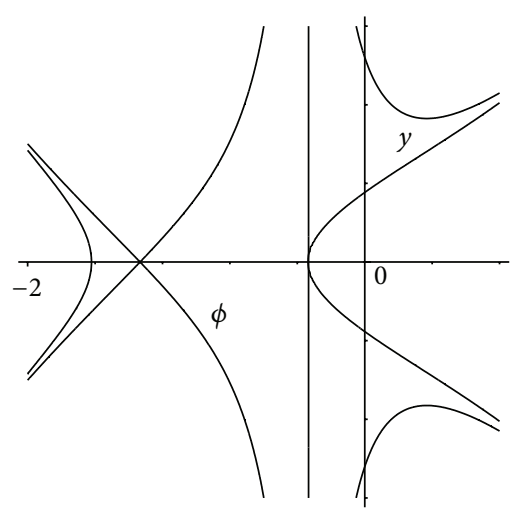

(j)

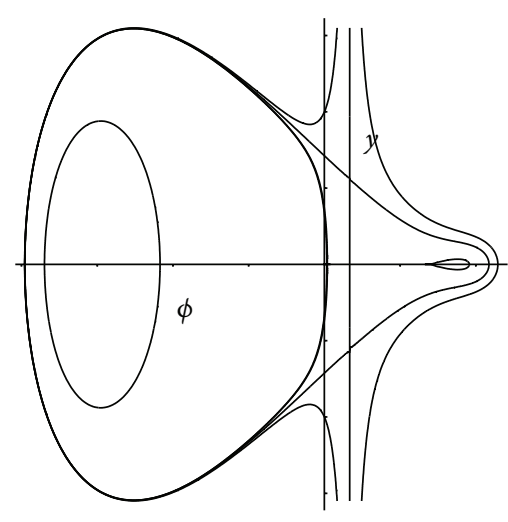

(e)

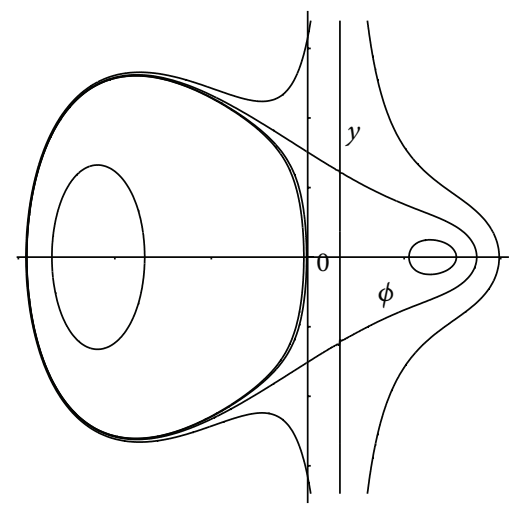

(h)

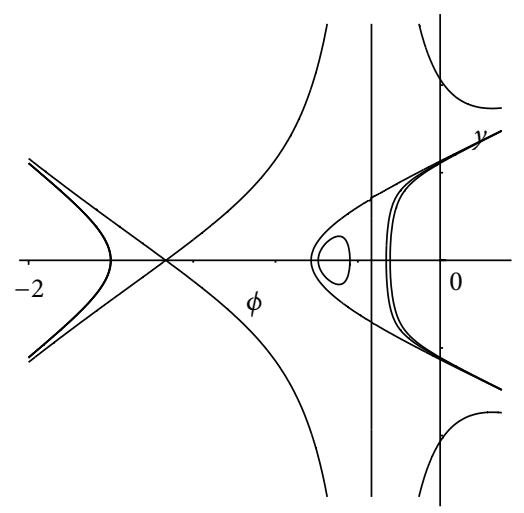

(k)

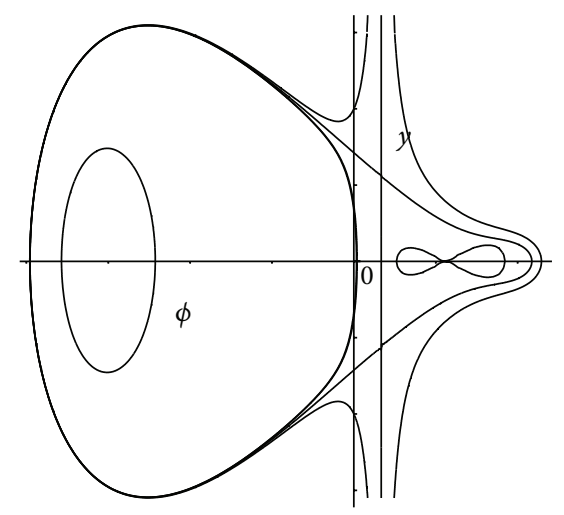

(f)

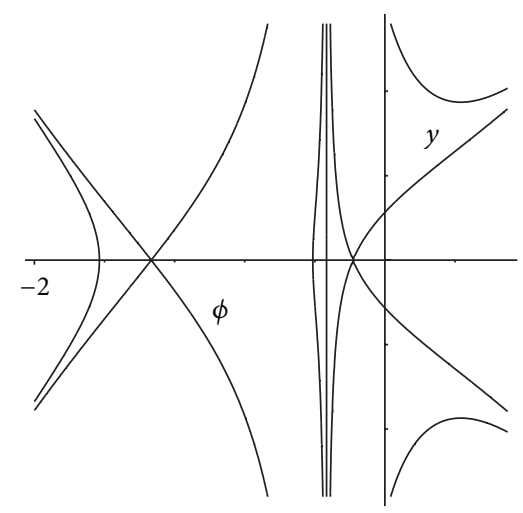

(i)

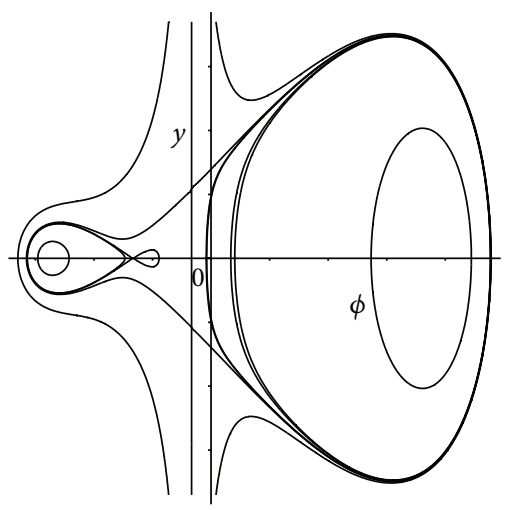

(1)

Figure 3: Continued. 


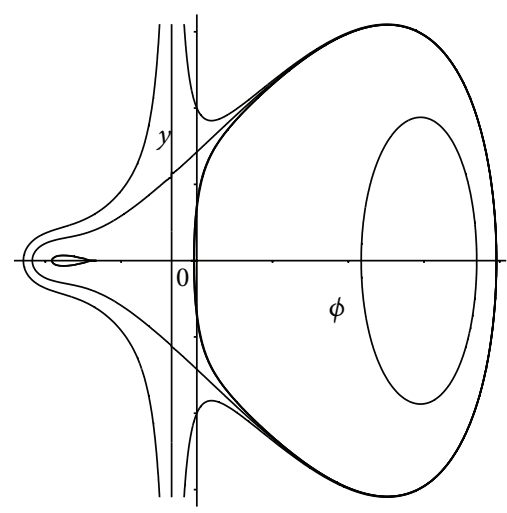

(m)

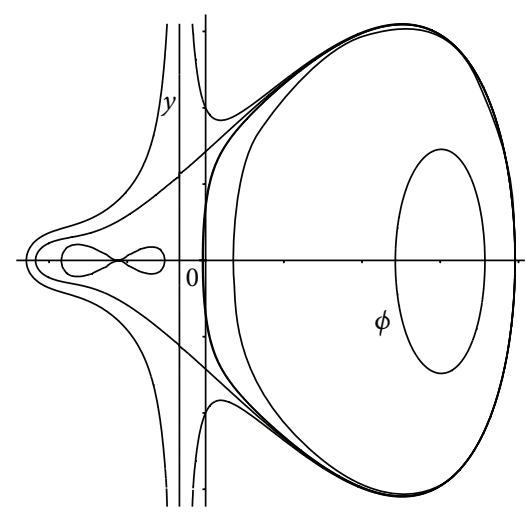

(n)

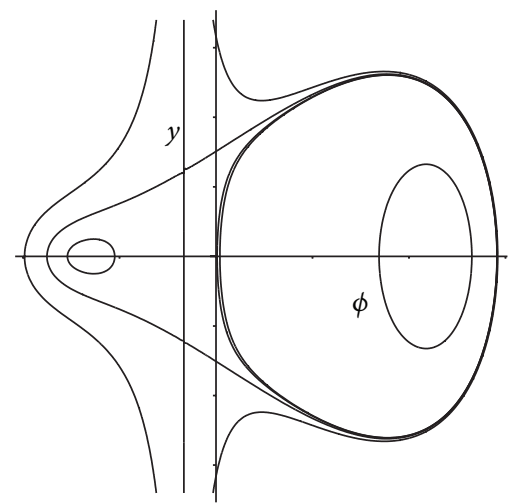

(p)

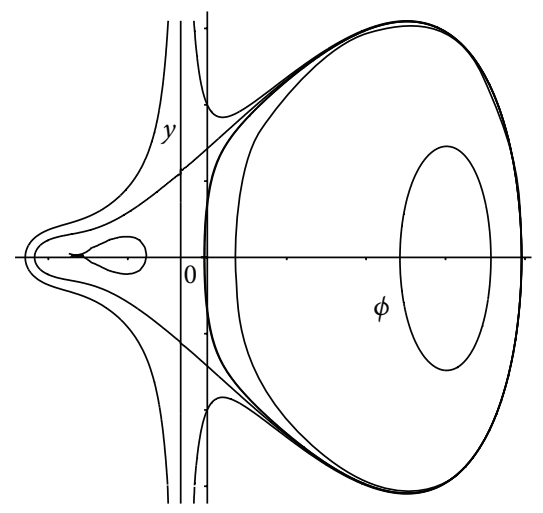

(o)

Figure 3: The bifurcation sets and phase portraits of system (13) when $k \neq 0$. (a) $(c, k) \in B_{1}$. (b) $(c, k) \in B_{2}$. (c) $(c, k) \in B_{3}$. (d) (c,k) $\in B_{4}$. (e) $(c, k) \in B_{5}$ (f) $(c, k) \in B_{6}$. (g) $(c, k) \in B_{7}$. (h) $(c, k) \in B_{8}$. (i) $(c, k) \in B_{9}$. (j) $(c, k) \in B_{10}$. (k) $(c, k) \in B_{11}$. (l) $(c, k) \in B_{12}$. (m) $(c, k) \in B_{13}$. (n) $(c, k) \in B_{14}$. (o) $(c, k) \in B_{15}$. (p) $(c, k) \in B_{16}$.

Completing the above integral, we can get the parametric representation of solitary wave solution as follows:

$$
\begin{gathered}
u(x, t)=\phi(x-c t)+\frac{4}{3} c, \\
\rho(x, t)=\left(\phi(x-c t)+\frac{1}{3} c\right)^{2} .
\end{gathered}
$$

$\phi$ is confirmed by

$$
\begin{aligned}
\phi(\chi)= & \frac{\beta_{2}\left(\beta_{3}-\beta_{1}\right)+\beta_{3}\left(\beta_{1}-\beta_{2}\right) \operatorname{sn}^{2}(\omega \chi, m)}{\left(\beta_{3}-\beta_{1}\right)+\left(\beta_{1}-\beta_{2}\right) \operatorname{sn}^{2}(\omega \chi, m)}, \\
\xi(\chi)=\widetilde{\Omega} & {\left[\left(\alpha^{2}-\alpha_{1}^{2}\right) \Pi\left(\arcsin (\operatorname{sn}(\omega \chi, m)), \alpha^{2}, m\right)\right.} \\
& \left.+\omega\left(\alpha_{1}^{2}-\frac{\alpha^{2}\left(\beta_{2}-\phi_{1}\right)}{\phi_{s}-\phi_{1}}\right) \chi\right],
\end{aligned}
$$

where $\widetilde{\Omega}=\left(\left(\phi_{s}-\phi_{1}\right) / \omega \alpha^{2}\left(\beta_{2}-\phi_{1}\right)\right) \sqrt{(6 / k)}, \alpha^{2}=$ $\left(\phi_{1}-\beta_{3}\right)\left(\beta_{1}-\beta_{2}\right) /\left(\phi_{1}-\beta_{2}\right)\left(\beta_{1}-\beta_{3}\right), \alpha_{1}^{2}=\left(\beta_{1}-\right.$ $\left.\beta_{2}\right) /\left(\beta_{1}-\beta_{3}\right), \omega=(1 / 2) \sqrt{\left(\beta_{1}-\beta_{3}\right)\left(\beta_{2}-\beta_{4}\right)}, m=$ $\sqrt{\left(\beta_{1}-\beta_{2}\right)\left(\beta_{3}-\beta_{4}\right) /\left(\beta_{1}-\beta_{3}\right)\left(\beta_{2}-\beta_{4}\right)}, \Pi(\cdot, \cdot, \cdot)$ is Legendre's incomplete elliptic integral of the third kind, $\operatorname{sn}(\cdot, \cdot)$ is the Jacobian elliptic function (see [11]), and $\chi$ is a new variable.
Substituting (27) into the $d \phi / d \xi=y$ and integrating it along the homoclinic orbit yield

$$
\int_{\phi}^{\beta_{1}} \frac{\left(s-\phi_{s}\right) d s}{\left(s-\phi_{1}\right) \sqrt{\left(\beta_{1}-s\right)\left(s-\beta_{2}\right)\left(s-\beta_{3}\right)\left(s-\beta_{4}\right)}}=\sqrt{\frac{k}{6}}|\xi| .
$$

Completing the above integral, we can get the parametric representation of solitary wave solution as follows:

$$
\begin{gathered}
u(x, t)=\phi(x-c t)+\frac{4}{3} c, \\
\rho(x, t)=\left(\phi(x-c t)+\frac{1}{3} c\right)^{2} .
\end{gathered}
$$

$\phi$ is confirmed by

$$
\begin{aligned}
\phi(\chi)= & \frac{\beta_{1}\left(\beta_{2}-\beta_{4}\right)+\beta_{4}\left(\beta_{1}-\beta_{2}\right) \operatorname{sn}^{2}(\omega \chi, m)}{\left(\beta_{2}-\beta_{4}\right)+\left(\beta_{1}-\beta_{2}\right) \operatorname{sn}^{2}(\omega \chi, m)}, \\
\xi(\chi)=\widetilde{\Omega} & {\left[\left(\alpha^{2}-\alpha_{1}^{2}\right) \Pi\left(\arcsin (\operatorname{sn}(\omega \chi, m)), \alpha^{2}, m\right)\right.} \\
& \left.+\omega\left(\alpha_{1}^{2}-\frac{\alpha^{2}\left(\beta_{1}-\phi_{1}\right)}{\phi_{s}-\phi_{1}}\right) \chi\right],
\end{aligned}
$$




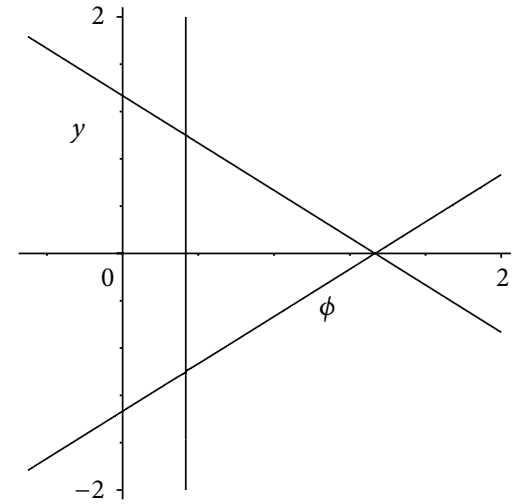

(a) $c<0, h_{*}=(16 / 9) c^{4}$

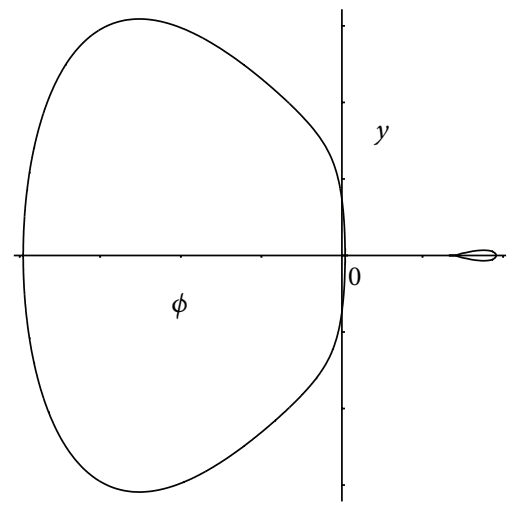

(d) $c<0, h_{*}=(128 / 243) c^{4}$

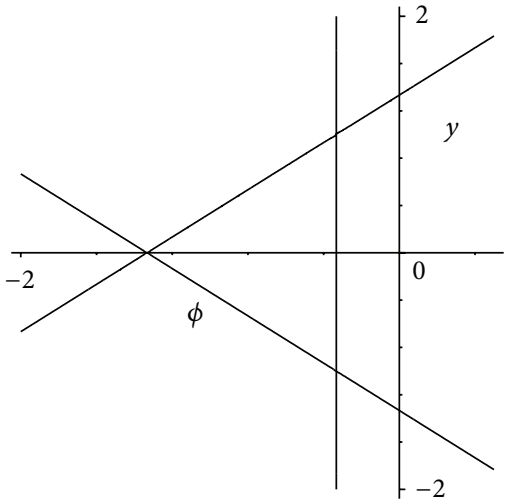

(b) $c>0, h_{*}=(16 / 9) c^{4}$

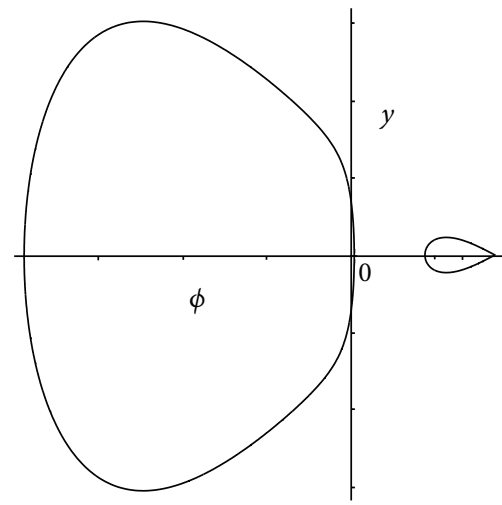

(e) $c<0, h_{*}=h_{2}$

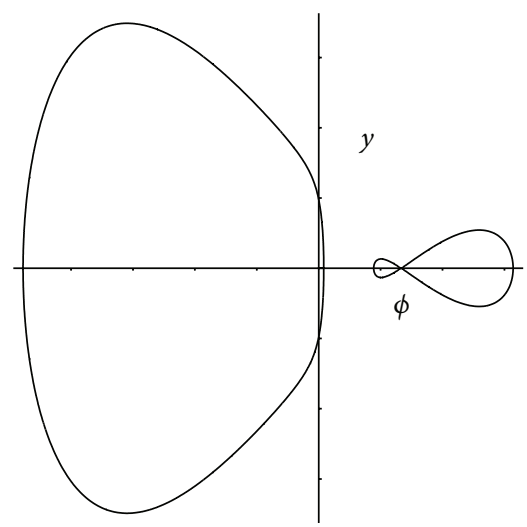

(c) $c<0, h_{*}=h_{1}$

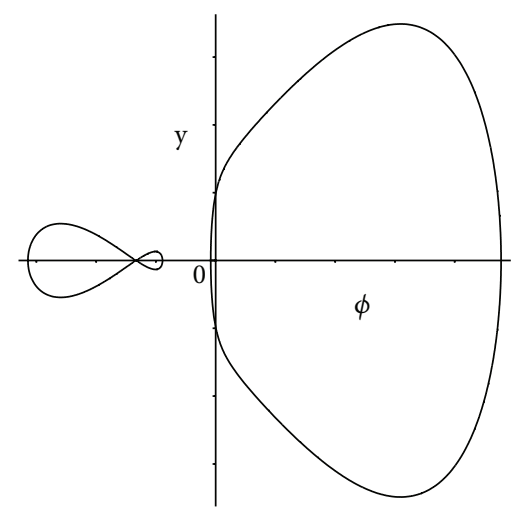

(f) $c>0, h_{*}=h_{1}$

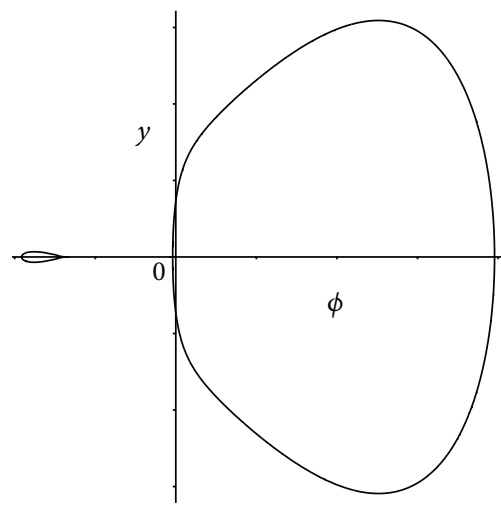

(g) $c>0, h_{*}=(128 / 243) c^{4}$

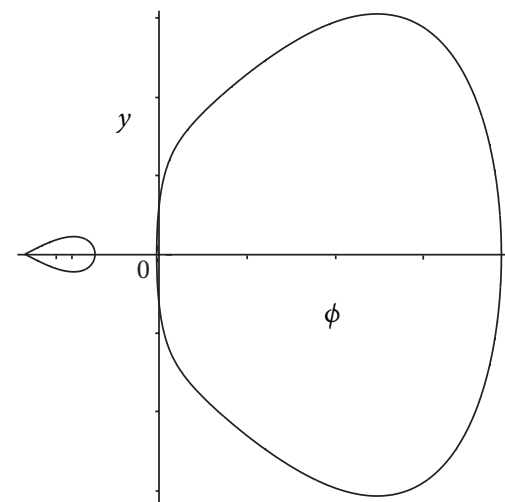

(h) $c>0, h_{*}=h_{2}$

FIgURE 4: The level curves of system (13) defined by $h=h_{*}$.

where $\widetilde{\Omega}=\left(\left(\phi_{s}-\phi_{1}\right) / \omega \alpha^{2}\left(\phi_{1}-\beta_{1}\right)\right) \sqrt{(6 / k)}, \alpha^{2}=$ $\left(\phi_{1}-\beta_{4}\right)\left(\beta_{1}-\beta_{2}\right) /\left(\beta_{1}-\phi_{1}\right)\left(\beta_{2}-\beta_{4}\right), \alpha_{1}^{2}=\left(\beta_{2}-\right.$ $\left.\beta_{1}\right) /\left(\beta_{2}-\beta_{4}\right), \omega=(1 / 2) \sqrt{\left(\beta_{1}-\beta_{3}\right)\left(\beta_{2}-\beta_{4}\right)}$, and $m=$ $\sqrt{\left(\beta_{1}-\beta_{2}\right)\left(\beta_{3}-\beta_{4}\right) /\left(\beta_{1}-\beta_{3}\right)\left(\beta_{2}-\beta_{4}\right)}$.

(ii) For the given $h=(128 / 243) c^{4}$ in Figure 3(e), the level curve is shown in Figure 4(d). From Figure 4(d), we see that there is a homoclinic orbit connecting with a cusp $\left(\phi_{1}, 0\right)$ of system (13) and passing point $\left(\beta_{1}, 0\right)$ when $(c, k) \in B_{5}$, and its expression is

$$
y= \pm \frac{1}{2 \sqrt{3} c}
$$

$$
\begin{array}{r}
\times \frac{\left(\phi-\phi_{1}\right) \sqrt{\left(\beta_{1}-\phi\right)\left(\phi-\phi_{1}\right)\left(\phi-\beta_{2}\right)\left(\phi-\beta_{3}\right)}}{\phi-\phi_{s}}, \\
\phi_{1}<\phi \leq \beta_{1},
\end{array}
$$

where $\beta_{1}, \beta_{2}$, and $\beta_{3}\left(\beta_{3}<\beta_{2}<\beta_{1}\right)$ are three real roots of

$$
\psi^{3}-2 c \psi^{2}-\frac{23}{3} c^{2} \psi-\frac{8}{27} c^{3}=0,
$$

which are obtained by the Cardan formula. For example, $\beta_{1} \approx 1.917558777, \beta_{2} \approx 0.03905297305, \beta_{3} \approx-3.956611750$ 
when $c=-1 ; \beta_{1} \approx 3.956611750, \beta_{2}-0.03905297305$, $\beta_{3} \approx-1.917558777$ when $c=1$; and $\beta_{1} \approx 0.9587793884$, $\beta_{2} \approx 0.01952648653, \beta_{3} \approx-1.978305875$ when $c=-0.5$.

Substituting (35) into the $d \phi / d \xi=y$ and integrating it along the homoclinic orbit yield

$$
\begin{aligned}
\int_{\phi}^{\beta_{1}} & \frac{\left(s-\phi_{s}\right) d s}{\left(s-\phi_{1}\right) \sqrt{\left(\beta_{1}-s\right)\left(s-\phi_{1}\right)\left(s-\beta_{2}\right)\left(s-\beta_{3}\right)}} \\
& =-\frac{1}{2 \sqrt{3} c}|\xi| .
\end{aligned}
$$

Completing the above integral, we can get the parametric representation of solitary wave solution as follows:

$$
\begin{gathered}
u(x, t)=\phi(x-c t)+\frac{4}{3} c, \\
\rho(x, t)=\left(\phi(x-c t)+\frac{1}{3} c\right)^{2} .
\end{gathered}
$$

$\phi$ is confirmed by

$$
\begin{gathered}
\phi(\chi)=\frac{\beta_{1}\left(\phi_{1}-\beta_{3}\right)+\beta_{3}\left(\beta_{1}-\phi_{1}\right) \operatorname{sn}^{2}(\omega \chi, m)}{\left(\phi_{1}-\beta_{3}\right)+\left(\beta_{1}-\phi_{1}\right) \operatorname{sn}^{2}(\omega \chi, m)}, \\
\xi(\chi)=\widetilde{\Omega}\left[\left(1-\alpha_{1}^{2}\right) \Pi(\arcsin (\operatorname{sn}(\omega \chi, m)), 1, m)\right. \\
\left.+\omega\left(\alpha_{1}^{2}-\frac{\beta_{1}-\beta_{3}}{\phi_{s}-\phi_{1}}\right) \chi\right]
\end{gathered}
$$

where $\widetilde{\Omega}=2 \sqrt{3}\left(\phi_{s}-\phi_{1}\right) c / \omega\left(\beta_{1}-\beta_{3}\right), \alpha_{1}^{2}=\left(\phi_{1}-\right.$ $\left.\beta_{1}\right) /\left(\phi_{1}-\beta_{3}\right), \omega=(1 / 2) \sqrt{\left(\beta_{1}-\beta_{2}\right)\left(\phi_{1}-\beta_{3}\right)}$, and $m=$ $\sqrt{\left(\beta_{1}-\phi_{1}\right)\left(\beta_{2}-\beta_{3}\right) /\left(\beta_{1}-\beta_{2}\right)\left(\phi_{1}-\beta_{3}\right)}$.

(iii) For the given $h=h_{2}$ in Figure 3(g), the level curve is shown in Figure 4(e). From Figure 4(e), we see that there is a homoclinic orbit connecting with a cusp $\left(\phi_{2}, 0\right)$ of system (13) and passing point $\left(\beta_{1}, 0\right)$ when $(c, k) \in B_{7}$, and its expression is

$$
\begin{aligned}
& y= \pm \sqrt{\frac{675 \Omega^{2}-9450 \Omega-84375}{24300 c^{2} \Omega}} \\
& \times \frac{\left(\phi_{2}-\phi\right) \sqrt{\left(\phi_{2}-\phi\right)\left(\phi-\beta_{1}\right)\left(\phi-\beta_{2}\right)\left(\phi-\beta_{3}\right)}}{\phi-\phi_{s}}, \\
& \beta_{1} \leq \phi<\phi_{2},
\end{aligned}
$$

where $\beta_{1}, \beta_{2}$, and $\beta_{3}\left(\beta_{3}<\beta_{2}<\beta_{1}\right)$ are three real roots of

$$
b_{0}+b_{1} \psi+b_{2} \psi^{2}-\psi^{3}=0
$$

which are obtained by the Cardan formula, and $b_{0}=$ $\left(c^{3}\left((486 \sqrt{14}-1663) \Omega^{2}-22375 \Omega+60750 \sqrt{14}+207875\right)\right) /$ $\left(675\left(\Omega^{2}-14 \Omega-125\right)\right), b_{1}=\left(c^{2}\left((1458 \sqrt{14}-6489) \Omega^{2}-\right.\right.$ $31950 \Omega+182250 \sqrt{14}+811125)) /\left(675\left(\Omega^{2}-14 \Omega-125\right)\right)$, and $b_{2}=\left(c\left((729 \sqrt{14}-4482) \Omega^{2}+5400 \Omega+91125 \sqrt{14}+\right.\right.$ $560250)) /\left(675\left(\Omega^{2}-14 \Omega-125\right)\right)$. For example, $\beta_{1} \approx$ 1.264234987, $\beta_{2} \approx 0.03471643922, \beta_{3} \approx-3.882082755$ when $c=-1 ; \beta_{1} \approx 6.321174982, \beta_{2} \approx 0.1735822117$, $\beta_{3} \approx-19.41041364$ when $c=-5$; and $\beta_{1} \approx 19.41041364$, $\beta_{2} \approx-0.1735822117, \beta_{3} \approx-6.321174982$ when $c=5$.

Substituting (40) into the $d \phi / d \xi=y$ and integrating it along the homoclinic orbit yield

$$
\begin{gathered}
\int_{\beta_{1}}^{\phi} \frac{\left(s-\phi_{s}\right) d s}{\left(s-\phi_{2}\right) \sqrt{\left(\phi_{2}-s\right)\left(s-\beta_{1}\right)\left(s-\beta_{2}\right)\left(s-\beta_{3}\right)}} \\
\quad=-\sqrt{\frac{675 \Omega^{2}-9450 \Omega-84375}{24300 c^{2} \Omega}}|\xi| .
\end{gathered}
$$

Completing the above integral, we can get the parametric representation of solitary wave solution as follows:

$$
\begin{gathered}
u(x, t)=\phi(x-c t)+\frac{4}{3} c, \\
\rho(x, t)=\left(\phi(x-c t)+\frac{1}{3} c\right)^{2} .
\end{gathered}
$$

$\phi$ is confirmed by

$$
\begin{gathered}
\phi(\chi)=\frac{\beta_{1}\left(\phi_{2}-\beta_{2}\right)+\beta_{2}\left(\beta_{1}-\phi_{2}\right) \operatorname{sn}^{2}(\omega \chi, m)}{\left(\phi_{2}-\beta_{2}\right)+\left(\beta_{1}-\phi_{2}\right) \operatorname{sn}^{2}(\omega \chi, m)}, \\
\xi(\chi)=\widetilde{\Omega}\left[\left(1-\alpha_{1}^{2}\right) \Pi(\arcsin (\operatorname{sn}(\omega \chi, m)), 1, m)\right. \\
\left.+\omega\left(\alpha_{1}^{2}-\frac{\phi_{2}-\beta_{1}}{\phi_{2}-\phi_{s}}\right) \chi\right]
\end{gathered}
$$

where $\widetilde{\Omega}=\left(\left(\phi_{2}-\phi_{s}\right) / \omega\left(\phi_{2}-\beta_{1}\right)\right)$ $\sqrt{24300 c^{2} \Omega /\left(675 \Omega^{2}-9450 \Omega-84375\right)}, \alpha_{1}^{2}=\left(\phi_{2}-\beta_{1}\right) /$ $\left(\phi_{2}-\beta_{2}\right), \omega=(1 / 2) \sqrt{\left(\phi_{2}-\beta_{2}\right)\left(\beta_{1}-\beta_{3}\right)}$, and $m=$ $\sqrt{\left(\phi_{2}-\beta_{1}\right)\left(\beta_{2}-\beta_{3}\right) /\left(\phi_{2}-\beta_{2}\right)\left(\beta_{1}-\beta_{3}\right)}$.

(iv) For the given $h=h_{1}$ in Figure 3(l), the level curve is shown in Figure 4(f). From Figure 4(f), we see that there is a homoclinic orbit connecting with a saddle point $\left(\phi_{1}, 0\right)$ of system (13) and passing point $\left(\beta_{4}, 0\right)$, and at the same time, there is a homoclinic orbit connecting with the saddle point $\left(\phi_{1}, 0\right)$ and passing point $\left(\beta_{3}, 0\right)$ when $(c, k) \in B_{12}$, and their expressions are

$$
\begin{aligned}
& y= \pm \sqrt{\frac{k}{6}} \\
& \times \frac{\left(\phi_{1}-\phi\right) \sqrt{\left(\beta_{1}-\phi\right)\left(\beta_{2}-\phi\right)\left(\beta_{3}-\phi\right)\left(\phi-\beta_{4}\right)}}{\phi_{s}-\phi}, \\
& \beta_{4} \leq \phi<\phi_{1},
\end{aligned}
$$




$$
\begin{aligned}
& y= \pm \sqrt{\frac{k}{6}} \\
& \times \frac{\left(\phi-\phi_{1}\right) \sqrt{\left(\beta_{1}-\phi\right)\left(\beta_{2}-\phi\right)\left(\beta_{3}-\phi\right)\left(\phi-\beta_{4}\right)}}{\phi-\phi_{s}}, \\
& \phi_{1}<\phi \leq \beta_{3},
\end{aligned}
$$

respectively, where $\beta_{1}, \beta_{2}, \beta_{3}$, and $\beta_{4}\left(\beta_{4}<\beta_{3}<\beta_{2}<\beta_{1}\right)$ are four real roots of (28).

Substituting (45) into the $d \phi / d \xi=y$ and integrating it along the homoclinic orbit yield

$$
\begin{aligned}
\int_{\beta_{4}}^{\phi} & \frac{\left(\phi_{s}-s\right) d s}{\left(\phi_{1}-s\right) \sqrt{\left(\beta_{1}-s\right)\left(\beta_{2}-s\right)\left(\beta_{3}-s\right)\left(s-\beta_{4}\right)}} \\
& =\sqrt{\frac{k}{6}}|\xi| .
\end{aligned}
$$

Completing the above integral, we can get the parametric representation of solitary wave solution as follows:

$$
\begin{gathered}
u(x, t)=\phi(x-c t)+\frac{4}{3} c, \\
\rho(x, t)=\left(\phi(x-c t)+\frac{1}{3} c\right)^{2} .
\end{gathered}
$$

$\phi$ is confirmed by

$$
\begin{aligned}
& \phi(\chi)= \frac{\beta_{4}\left(\beta_{1}-\beta_{3}\right)+\beta_{1}\left(\beta_{3}-\beta_{4}\right) \operatorname{sn}^{2}(\omega \chi, m)}{\left(\beta_{1}-\beta_{3}\right)+\left(\beta_{3}-\beta_{4}\right) \operatorname{sn}^{2}(\omega \chi, m)}, \\
& \xi(\chi)=\widetilde{\Omega}\left[\left(\alpha^{2}-\alpha_{1}^{2}\right) \Pi\left(\arcsin (\operatorname{sn}(\omega \chi, m)), \alpha^{2}, m\right)\right. \\
&\left.+\omega\left(\alpha_{1}^{2}-\frac{\alpha^{2}\left(\beta_{4}-\phi_{1}\right)}{\phi_{s}-\phi_{1}}\right) \chi\right],
\end{aligned}
$$

where $\widetilde{\Omega}=\left(\left(\phi_{s}-\phi_{1}\right) / \omega \alpha^{2}\left(\phi_{1}-\beta_{4}\right)\right) \sqrt{6 / k}, \alpha^{2}=$ $\left(\phi_{1}-\beta_{1}\right)\left(\beta_{4}-\beta_{3}\right) /\left(\phi_{1}-\beta_{4}\right)\left(\beta_{1}-\beta_{3}\right), \alpha_{1}^{2}=\left(\beta_{4}-\right.$ $\left.\beta_{3}\right) /\left(\beta_{1}-\beta_{3}\right), \omega=(1 / 2) \sqrt{\left(\beta_{1}-\beta_{3}\right)\left(\beta_{2}-\beta_{4}\right)}$, and $m=$ $\sqrt{\left(\beta_{1}-\beta_{2}\right)\left(\beta_{3}-\beta_{4}\right) /\left(\beta_{1}-\beta_{3}\right)\left(\beta_{2}-\beta_{4}\right)}$.

Substituting (46) into the $d \phi / d \xi=y$ and integrating it along the homoclinic orbit yield

$$
\begin{aligned}
& \int_{\phi}^{\beta_{3}} \frac{\left(s-\phi_{s}\right) d s}{\left(s-\phi_{1}\right) \sqrt{\left(\beta_{1}-s\right)\left(\beta_{2}-s\right)\left(\beta_{3}-s\right)\left(s-\beta_{4}\right)}} \\
& \quad=-\sqrt{\frac{k}{6}}|\xi| .
\end{aligned}
$$

Completing the above integral, we can get the parametric representation of solitary wave solution as follows:

$$
\begin{gathered}
u(x, t)=\phi(x-c t)+\frac{4}{3} c, \\
\rho(x, t)=\left(\phi(x-c t)+\frac{1}{3} c\right)^{2} .
\end{gathered}
$$

$\phi$ is confirmed by

$$
\begin{aligned}
\phi(\chi)=\frac{\beta_{3}\left(\beta_{4}-\beta_{2}\right)+\beta_{2}\left(\beta_{3}-\beta_{4}\right) \operatorname{sn}^{2}(\omega \chi, m)}{\left(\beta_{4}-\beta_{2}\right)+\left(\beta_{3}-\beta_{4}\right) \operatorname{sn}^{2}(\omega \chi, m)}, \\
\xi(\chi)=\widetilde{\Omega}\left[\left(\alpha^{2}-\alpha_{1}^{2}\right) \Pi\left(\arcsin (\operatorname{sn}(\omega \chi, m)), \alpha^{2}, m\right)\right. \\
\left.+\omega\left(\alpha_{1}^{2}-\frac{\alpha^{2}\left(\beta_{3}-\phi_{1}\right)}{\phi_{s}-\phi_{1}}\right) \chi\right],
\end{aligned}
$$

where $\widetilde{\Omega}=\left(\left(\phi_{1}-\phi_{s}\right) / \omega \alpha^{2}\left(\phi_{1}-\beta_{3}\right)\right) \sqrt{6 / k}, \alpha^{2}=$ $\left(\phi_{1}-\beta_{2}\right)\left(\beta_{3}-\beta_{4}\right) /\left(\phi_{1}-\beta_{3}\right)\left(\beta_{2}-\beta_{4}\right), \alpha_{1}^{2}=\left(\beta_{3}-\right.$ $\left.\beta_{4}\right) /\left(\beta_{2}-\beta_{4}\right), \omega=(1 / 2) \sqrt{\left(\beta_{1}-\beta_{3}\right)\left(\beta_{2}-\beta_{4}\right)}$, and $m=$ $\sqrt{\left(\beta_{1}-\beta_{2}\right)\left(\beta_{3}-\beta_{4}\right) /\left(\beta_{1}-\beta_{3}\right)\left(\beta_{2}-\beta_{4}\right)}$.

(v) For the given $h=(128 / 243) c^{4}$ in Figure 3(m), the level curve is shown in Figure 4(g). From Figure $4(\mathrm{~g})$, we see that there is a homoclinic orbit connecting with a cusp $\left(\phi_{1}, 0\right)$ of system (13) and passing point $\left(\beta_{3}, 0\right)$ when $(c, k) \in B_{13}$, and its expression is

$$
\begin{aligned}
y= & \pm \frac{1}{2 \sqrt{3} c} \\
& \times \frac{\left(\phi_{1}-\phi\right) \sqrt{\left(\beta_{1}-\phi\right)\left(\beta_{2}-\phi\right)\left(\phi_{1}-\phi\right)\left(\phi-\beta_{3}\right)}}{\phi_{s}-\phi},
\end{aligned}
$$$$
\beta_{3} \leq \phi<\phi_{1}
$$

where $\beta_{1}, \beta_{2}$, and $\beta_{3}\left(\beta_{3}<\beta_{2}<\beta_{1}\right)$ are three real roots of (36).

Substituting (53) into the $d \phi / d \xi=y$ and integrating it along the homoclinic orbit yield

$$
\begin{aligned}
\int_{\beta_{3}}^{\phi} & \frac{\left(\phi_{s}-s\right) d s}{\left(\phi_{1}-s\right) \sqrt{\left(\beta_{1}-s\right)\left(\beta_{2}-s\right)\left(\phi_{1}-s\right)\left(s-\beta_{3}\right)}} \\
& =\frac{1}{2 \sqrt{3} c}|\xi| .
\end{aligned}
$$

Completing the above integral, we can get the parametric representation of solitary wave solution as follows:

$$
\begin{gathered}
u(x, t)=\phi(x-c t)+\frac{4}{3} c, \\
\rho(x, t)=\left(\phi(x-c t)+\frac{1}{3} c\right)^{2} .
\end{gathered}
$$

$\phi$ is confirmed by

$$
\begin{gathered}
\phi(\chi)=\frac{\beta_{3}\left(\phi_{1}-\beta_{1}\right)+\beta_{1}\left(\beta_{3}-\phi_{1}\right) \operatorname{sn}^{2}(\omega \chi, m)}{\left(\phi_{1}-\beta_{1}\right)+\left(\beta_{3}-\phi_{1}\right) \operatorname{sn}^{2}(\omega \chi, m)}, \\
\xi(\chi)=\widetilde{\Omega}\left[\left(1-\alpha_{1}^{2}\right) \Pi(\arcsin (\operatorname{sn}(\omega \chi, m)), 1, m)\right. \\
\left.+\omega\left(\alpha_{1}^{2}-\frac{\beta_{3}-\phi_{1}}{\phi_{s}-\phi_{1}}\right) \chi\right],
\end{gathered}
$$


where $\widetilde{\Omega}=2 \sqrt{3}\left(\phi_{s}-\phi_{1}\right) c / \omega\left(\phi_{1}-\beta_{3}\right), \alpha_{1}^{2}=\left(\phi_{1}-\beta_{3}\right) /$ $\left(\phi_{1}-\beta_{1}\right), \omega=(1 / 2) \sqrt{\left(\beta_{2}-\beta_{3}\right)\left(\beta_{1}-\phi_{1}\right)}$, and $m=$ $\sqrt{\left(\beta_{3}-\phi_{1}\right)\left(\beta_{2}-\beta_{1}\right) /\left(\beta_{3}-\beta_{2}\right)\left(\phi_{1}-\beta_{1}\right)}$.

(vi) For the given $h=h_{2}$ in Figure 3(o), the level curve is shown in Figure 4(h). From Figure 4(h), we see that there is a homoclinic orbit connecting with a cusp $\left(\phi_{2}, 0\right)$ of system (13) and passing point $\left(\beta_{3}, 0\right)$ when $(c, k) \in B_{15}$, and its expression is

$$
\begin{aligned}
& y= \pm \sqrt{\frac{675 \Omega^{2}-9450 \Omega-84375}{24300 c^{2} \Omega}} \\
& \times \frac{\left(\phi-\phi_{2}\right) \sqrt{\left(\phi-\phi_{2}\right)\left(\beta_{1}-\phi\right)\left(\phi-\beta_{2}\right)\left(\phi-\beta_{3}\right)}}{\phi-\phi_{s}}, \\
& \phi_{2}<\phi \leq \beta_{3},
\end{aligned}
$$

where $\beta_{1}, \beta_{2}$, and $\beta_{3}\left(\beta_{3}<\beta_{2}<\beta_{1}\right)$ are three real roots of (41).

Substituting (57) into the $d \phi / d \xi=y$ and integrating it along the homoclinic orbit yield

$$
\begin{gathered}
\int_{\phi}^{\beta_{3}} \frac{\left(s-\phi_{s}\right) d s}{\left(s-\phi_{2}\right) \sqrt{\left(\beta_{1}-s\right)\left(\beta_{2}-s\right)\left(\beta_{3}-s\right)\left(s-\phi_{2}\right)}} \\
\quad=-\sqrt{\frac{675 \Omega^{2}-9450 \Omega-84375}{24300 c^{2} \Omega}}|\xi| .
\end{gathered}
$$

Completing the above integral, we can get the parametric representation of solitary wave solution as follows:

$$
\begin{gathered}
u(x, t)=\phi(x-c t)+\frac{4}{3} c, \\
\rho(x, t)=\left(\phi(x-c t)+\frac{1}{3} c\right)^{2} .
\end{gathered}
$$

$\phi$ is confirmed by

$$
\begin{gathered}
\phi(\chi)=\frac{\beta_{3}\left(\phi_{2}-\beta_{2}\right)+\beta_{2}\left(\beta_{3}-\phi_{2}\right) \operatorname{sn}^{2}(\omega \chi, m)}{\left(\phi_{2}-\beta_{2}\right)+\left(\beta_{3}-\phi_{2}\right) \operatorname{sn}^{2}(\omega \chi, m)}, \\
\xi(\chi)=\widetilde{\Omega}\left[\left(1-\alpha_{1}^{2}\right) \Pi(\arcsin (\operatorname{sn}(\omega \chi, m)), 1, m)\right. \\
\left.+\omega\left(\alpha_{1}^{2}-\frac{\phi_{2}-\beta_{3}}{\phi_{2}-\phi_{s}}\right) \chi\right]
\end{gathered}
$$

where $\widetilde{\Omega}=\left(\left(\phi_{2}-\phi_{s}\right) / \omega\left(\phi_{2}-\beta_{3}\right)\right)$ $\sqrt{24300 c^{2} \Omega /\left(675 \Omega^{2}-9450 \Omega-84375\right)}, \quad \alpha_{1}^{2}=\left(\phi_{2}-\right.$ $\left.\beta_{3}\right) /\left(\phi_{2}-\beta_{2}\right), \omega=(1 / 2) \sqrt{\left(\phi_{2}-\beta_{2}\right)\left(\beta_{3}-\beta_{1}\right)}$, and $m=$ $\sqrt{\left(\phi_{2}-\beta_{3}\right)\left(\beta_{2}-\beta_{1}\right) /\left(\phi_{2}-\beta_{2}\right)\left(\beta_{3}-\beta_{1}\right)}$.

3.3. Exact Parametric Representations of Smooth Periodic Wave Solutions. (i) For the given $h=h_{1}$ in Figure 3(d), the level curve is shown in Figure 4(c). From Figure 4(c), we see that there is one periodic orbit passing points $\left(\beta_{3}, 0\right)$ and $\left(\beta_{4}, 0\right)$ when $(c, k) \in B_{4}$, and its expression is

$$
\begin{aligned}
& y= \pm \sqrt{\frac{k}{6}} \\
& \times \frac{\left(\phi-\phi_{1}\right) \sqrt{\left(\beta_{1}-\phi\right)\left(\beta_{2}-\phi\right)\left(\beta_{3}-\phi\right)\left(\phi-\beta_{4}\right)}}{\phi-\phi_{s}}, \\
& \beta_{4} \leq \phi \leq \beta_{3},
\end{aligned}
$$

where $\beta_{1}, \beta_{2}, \beta_{3}$, and $\beta_{4}\left(\beta_{4}<\beta_{3}<\beta_{2}<\beta_{1}\right)$ are four real roots of (28).

Substituting (61) into the $d \phi / d \xi=y$ and integrating it along the periodic orbit yield

$$
\begin{aligned}
\int_{\phi}^{\beta_{3}} & \frac{\left(\phi_{s}-s\right) d s}{\left(\phi_{1}-s\right) \sqrt{\left(\beta_{1}-s\right)\left(\beta_{2}-s\right)\left(\beta_{3}-s\right)\left(s-\beta_{4}\right)}} \\
& =\sqrt{\frac{k}{6}}|\xi| .
\end{aligned}
$$

Completing the above integral, we can get the parametric representation of smooth periodic wave solution as follows:

$$
\begin{gathered}
u(x, t)=\phi(x-c t)+\frac{4}{3} c, \\
\rho(x, t)=\left(\phi(x-c t)+\frac{1}{3} c\right)^{2} .
\end{gathered}
$$

$\phi$ is confirmed by

$$
\begin{aligned}
& \phi(\chi)= \frac{\beta_{3}\left(\beta_{4}-\beta_{2}\right)+\beta_{2}\left(\beta_{3}-\beta_{4}\right) \operatorname{sn}^{2}(\omega \chi, m)}{\left(\beta_{4}-\beta_{2}\right)+\left(\beta_{3}-\beta_{4}\right) \operatorname{sn}^{2}(\omega \chi, m)}, \\
& \xi(\chi)=\widetilde{\Omega} {\left[\left(\alpha^{2}-\alpha_{1}^{2}\right) \Pi\left(\arcsin (\operatorname{sn}(\omega \chi, m)), \alpha^{2}, m\right)\right.} \\
&\left.+\omega\left(\alpha_{1}^{2}-\frac{\alpha^{2}\left(\beta_{3}-\phi_{1}\right)}{\phi_{s}-\phi_{1}}\right) \chi\right],
\end{aligned}
$$

where $\widetilde{\Omega}=\left(\left(\phi_{s}-\phi_{1}\right) / \omega \alpha^{2}\left(\phi_{1}-\beta_{3}\right)\right) \sqrt{6 / k}, \alpha^{2}=\left(\phi_{1}-\beta_{2}\right)$ $\left(\beta_{3}-\beta_{4}\right) /\left(\phi_{1}-\beta_{3}\right)\left(\beta_{2}-\beta_{4}\right), \alpha_{1}^{2}=\left(\beta_{3}-\beta_{4}\right) /\left(\beta_{2}-\right.$ $\left.\beta_{4}\right), \omega=(1 / 2) \sqrt{\left(\beta_{1}-\beta_{3}\right)\left(\beta_{2}-\beta_{4}\right)}$, and $m=$ $\sqrt{\left(\beta_{1}-\beta_{2}\right)\left(\beta_{3}-\beta_{4}\right) /\left(\beta_{1}-\beta_{3}\right)\left(\beta_{2}-\beta_{4}\right)}$.

(ii) For the given $h=(128 / 243) c^{4}$ in Figure 3(e), the level curve is shown in Figure 4(d). From Figure 4(d), we see that there is one periodic orbit passing points $\left(\beta_{2}, 0\right)$ and $\left(\beta_{3}, 0\right)$ when $(c, k) \in B_{5}$, and its expression is

$$
\begin{aligned}
y= \pm & \frac{1}{2 \sqrt{3} c} \\
& \times \frac{\left(\phi_{1}-\phi\right) \sqrt{\left(\beta_{1}-\phi\right)\left(\phi_{1}-\phi\right)\left(\beta_{2}-\phi\right)\left(\phi-\beta_{3}\right)}}{\phi_{s}-\phi}, \\
\beta_{3} \leq \phi \leq \beta_{2}, &
\end{aligned}
$$


where $\beta_{1}, \beta_{2}$, and $\beta_{3}\left(\beta_{3}<\beta_{2}<\beta_{1}\right)$ are three real roots of (36).

Substituting (65) into the $d \phi / d \xi=y$ and integrating it along the periodic orbit yield

$$
\begin{aligned}
\int_{\phi}^{\beta_{2}} & \frac{\left(\phi_{s}-s\right) d s}{\left(\phi_{1}-s\right) \sqrt{\left(\beta_{1}-s\right)\left(\phi_{1}-s\right)\left(\beta_{2}-s\right)\left(s-\beta_{3}\right)}} \\
& =-\frac{1}{2 \sqrt{3} c}|\xi| .
\end{aligned}
$$

Completing the above integral, we can get the parametric representation of smooth periodic wave solution as follows:

$$
\begin{gathered}
u(x, t)=\phi(x-c t)+\frac{4}{3} c \\
\rho(x, t)=\left(\phi(x-c t)+\frac{1}{3} c\right)^{2} .
\end{gathered}
$$

$\phi$ is confirmed by

$$
\phi(\chi)=\frac{\beta_{2}\left(\beta_{3}-\phi_{1}\right)+\phi_{1}\left(\beta_{2}-\beta_{3}\right) \operatorname{sn}^{2}(\omega \chi, m)}{\left(\beta_{3}-\phi_{1}\right)+\left(\beta_{2}-\beta_{3}\right) \operatorname{sn}^{2}(\omega \chi, m)},
$$

$\xi(\chi)$

$$
\begin{aligned}
=\widetilde{\Omega}[ & \left(m^{2}-\alpha^{2}\right) \mathrm{F}(\arcsin (\operatorname{sn}(\omega \chi, m)), m) \\
& \left.\quad+\alpha^{2} \mathrm{E}(\arcsin (\operatorname{sn}(\omega \chi, m)), m)-\frac{\omega m^{2}\left(\phi_{1}-\beta_{2}\right)}{\phi_{1}-\phi_{s}} \chi\right],
\end{aligned}
$$

where $\widetilde{\Omega}=\left(2 \sqrt{3}\left(\phi_{1}-\phi_{s}\right) c\right) / \omega m^{2}\left(\phi_{1}-\beta_{2}\right), \alpha^{2}=$ $\left(\beta_{2}-\beta_{3}\right) /\left(\phi_{1}-\beta_{3}\right), \omega=(1 / 2) \sqrt{\left(\beta_{1}-\beta_{2}\right)\left(\phi_{1}-\beta_{3}\right)}, m=$ $\sqrt{\left(\beta_{1}-\phi_{1}\right)\left(\beta_{2}-\beta_{3}\right) /\left(\beta_{1}-\beta_{2}\right)\left(\phi_{1}-\beta_{3}\right)}$, and $\mathrm{F}(\cdot, \cdot)$ and $\mathrm{E}(\cdot, \cdot)$ are Legendre's incomplete elliptic integrals of the first and second kinds, respectively (see [11]).

(iii) For the given $h=h_{2}$ in Figure 3(g), the level curve is shown in Figure 4(e). From Figure 4(e), we see that there is one periodic orbit passing points $\left(\beta_{2}, 0\right)$ and $\left(\beta_{3}, 0\right)$ when $(c, k) \in B_{7}$, and its expression is

$$
\begin{aligned}
& y= \pm \sqrt{\frac{675 \Omega^{2}-9450 \Omega-84375}{24300 c^{2} \Omega}} \\
& \times \frac{\left(\phi_{2}-\phi\right) \sqrt{\left(\phi_{2}-\phi\right)\left(\beta_{1}-\phi\right)\left(\beta_{2}-\phi\right)\left(\phi-\beta_{3}\right)}}{\phi_{s}-\phi}, \\
& \beta_{3} \leq \phi \leq \beta_{2},
\end{aligned}
$$

where $\beta_{1}, \beta_{2}$, and $\beta_{3}\left(\beta_{3}<\beta_{2}<\beta_{1}\right)$ are three real roots of (41).
Substituting (69) into the $d \phi / d \xi=y$ and integrating it along the periodic orbit yield

$$
\begin{gathered}
\int_{\phi}^{\beta_{2}} \frac{\left(\phi_{s}-s\right) d s}{\left(\phi_{2}-s\right) \sqrt{\left(\phi_{2}-s\right)\left(\beta_{1}-s\right)\left(\beta_{2}-s\right)\left(s-\beta_{3}\right)}} \\
\quad=\sqrt{\frac{675 \Omega^{2}-9450 \Omega-84375}{24300 c^{2} \Omega}}|\xi| .
\end{gathered}
$$

Completing the above integral, we can get the parametric representation of smooth periodic wave solution as follows:

$$
\begin{gathered}
u(x, t)=\phi(x-c t)+\frac{4}{3} c, \\
\rho(x, t)=\left(\phi(x-c t)+\frac{1}{3} c\right)^{2} .
\end{gathered}
$$

$\phi$ is confirmed by

$$
\begin{aligned}
\phi(\chi)= & \frac{\beta_{2}\left(\beta_{3}-\beta_{1}\right)+\beta_{1}\left(\beta_{2}-\beta_{3}\right) \operatorname{sn}^{2}(\omega \chi, m)}{\left(\beta_{3}-\beta_{1}\right)+\left(\beta_{2}-\beta_{3}\right) \operatorname{sn}^{2}(\omega \chi, m)}, \\
\xi(\chi) & \\
=\widetilde{\Omega} & {\left[\left(m^{2}-\alpha_{1}^{2}\right) \Pi\left(\arcsin (\operatorname{sn}(\omega \chi, m)), m^{2}, m\right)\right.} \\
& \left.+\omega\left(\alpha_{1}^{2}-\frac{m^{2}\left(\phi_{2}-\beta_{2}\right)}{\phi_{2}-\phi_{s}}\right) \chi\right],
\end{aligned}
$$

where $\widetilde{\Omega}=\left(\left(\phi_{s}-\phi_{2}\right) / \omega m^{2}\left(\phi_{2}-\beta_{2}\right)\right)$ $\sqrt{24300 c^{2} \Omega /\left(675 \Omega^{2}-9450 \Omega-84375\right)}, \alpha_{1}^{2}=\left(\beta_{2}-\beta_{3}\right) /$ $\left(\beta_{1}-\beta_{3}\right), \omega=(1 / 2) \sqrt{\left(\phi_{2}-\beta_{2}\right)\left(\beta_{1}-\beta_{3}\right)}$, and $m=$ $\sqrt{\left(\phi_{2}-\beta_{1}\right)\left(\beta_{2}-\beta_{3}\right) /\left(\phi_{2}-\beta_{2}\right)\left(\beta_{1}-\beta_{3}\right)}$.

(iv) For the given $h=h_{1}$ in Figure 3(1), the level curve is shown in Figure 4(f). From Figure 4(f), we see that there is one periodic orbit passing points $\left(\beta_{1}, 0\right)$ and $\left(\beta_{2}, 0\right)$ when $(c, k) \in B_{12}$, and its expression is

$$
\begin{array}{r}
y= \pm \sqrt{\frac{k}{6}} \frac{\left(\phi-\phi_{1}\right) \sqrt{\left(\beta_{1}-\phi\right)\left(\phi-\beta_{2}\right)\left(\phi-\beta_{3}\right)\left(\phi-\beta_{4}\right)}}{\phi-\phi_{s}}, \\
\beta_{2} \leq \phi \leq \beta_{1},
\end{array}
$$

where $\beta_{1}, \beta_{2}, \beta_{3}$, and $\beta_{4}\left(\beta_{4}<\beta_{3}<\beta_{2}<\beta_{1}\right)$ are four real roots of (28).

Substituting (73) into the $d \phi / d \xi=y$ and integrating it along the periodic orbit yield

$$
\begin{aligned}
\int_{\phi}^{\beta_{1}} & \frac{\left(s-\phi_{s}\right) d s}{\left(s-\phi_{1}\right) \sqrt{\left(\beta_{1}-s\right)\left(s-\beta_{2}\right)\left(s-\beta_{3}\right)\left(s-\beta_{4}\right)}} \\
& =\sqrt{\frac{k}{6}}|\xi| .
\end{aligned}
$$


Completing the above integral, we can get the parametric representation of smooth periodic wave solution which is the same as (33).

(v) For the given $h=(128 / 243) c^{4}$ in Figure 3(m), the level curve is shown in Figure 4(g). From Figure 4(g), we see that there is one periodic orbit passing points $\left(\beta_{1}, 0\right)$ and $\left(\beta_{2}, 0\right)$ when $(c, k) \in B_{13}$, and its expression is

$$
\begin{aligned}
& y= \pm \frac{1}{2 \sqrt{3} c} \\
& \times \frac{\left(\phi-\phi_{1}\right) \sqrt{\left(\beta_{1}-\phi\right)\left(\phi-\beta_{2}\right)\left(\phi-\phi_{1}\right)\left(\phi-\beta_{3}\right)}}{\phi-\phi_{s}}, \\
& \beta_{2} \leq \phi \leq \beta_{1},
\end{aligned}
$$

where $\beta_{1}, \beta_{2}$, and $\beta_{3}\left(\beta_{3}<\beta_{2}<\beta_{1}\right)$ are three real roots of (36).

Substituting (75) into the $d \phi / d \xi=y$ and integrating it along the periodic orbit yield

$$
\begin{aligned}
\int_{\phi}^{\beta_{1}} & \frac{\left(s-\phi_{s}\right) d s}{\left(s-\phi_{1}\right) \sqrt{\left(\beta_{1}-s\right)\left(s-\beta_{2}\right)\left(s-\phi_{1}\right)\left(s-\beta_{3}\right)}} \\
& =\frac{1}{2 \sqrt{3} c}|\xi| .
\end{aligned}
$$

Completing the above integral, we can get the parametric representation of smooth periodic wave solution as follows:

$$
\begin{gathered}
u(x, t)=\phi(x-c t)+\frac{4}{3} c, \\
\rho(x, t)=\left(\phi(x-c t)+\frac{1}{3} c\right)^{2} .
\end{gathered}
$$

$\phi$ is confirmed by

$$
\begin{gathered}
\phi(\chi)=\frac{\beta_{1}\left(\beta_{2}-\beta_{3}\right)+\beta_{3}\left(\beta_{1}-\beta_{3}\right) \operatorname{sn}^{2}(\omega \chi, m)}{\left(\beta_{2}-\beta_{3}\right)+\left(\beta_{1}-\beta_{3}\right) \operatorname{sn}^{2}(\omega \chi, m)}, \\
\xi(\chi)=\widetilde{\Omega}\left[\left(m^{2}-\alpha_{1}^{2}\right) \Pi\left(\arcsin (\operatorname{sn}(\omega \chi, m)), m^{2}, m\right)\right. \\
\left.+\omega\left(\alpha_{1}^{2}-\frac{m^{2}\left(\phi_{1}-\beta_{1}\right)}{\phi_{1}-\phi_{s}}\right) \chi\right],
\end{gathered}
$$

where $\widetilde{\Omega}=2 \sqrt{3}\left(\phi_{1}-\phi_{s}\right) c / \omega m^{2}\left(\beta_{1}-\phi_{1}\right), \alpha_{1}^{2}=\left(\beta_{2}-\right.$ $\left.\beta_{1}\right) /\left(\beta_{2}-\beta_{3}\right), \omega=(1 / 2) \sqrt{\left(\beta_{1}-\phi_{1}\right)\left(\beta_{2}-\beta_{3}\right)}$, and $m=$ $\sqrt{\left(\beta_{1}-\beta_{2}\right)\left(\phi_{1}-\beta_{3}\right) /\left(\beta_{1}-\phi_{1}\right)\left(\beta_{2}-\beta_{3}\right)}$.

(vi) For the given $h=h_{2}$ in Figure 3(o), the level curve is shown in Figure 4(h). From Figure 4(h), we see that there is one periodic orbit passing points $\left(\beta_{1}, 0\right)$ and $\left(\beta_{2}, 0\right)$ when $(c, k) \in B_{15}$, and its expression is

$$
\begin{aligned}
& y= \pm \sqrt{\frac{675 \Omega^{2}-9450 \Omega-84375}{24300 c^{2} \Omega}} \\
& \times \frac{\left(\phi-\phi_{2}\right) \sqrt{\left(\beta_{1}-\phi\right)\left(\phi-\beta_{2}\right)\left(\phi-\beta_{3}\right)\left(\phi-\phi_{2}\right)}}{\phi-\phi_{s}}, \\
& \beta_{2} \leq \phi \leq \beta_{1},
\end{aligned}
$$

where $\beta_{1}, \beta_{2}$, and $\beta_{3}\left(\beta_{3}<\beta_{2}<\beta_{1}\right)$ are three real roots of (41).

Substituting (79) into the $d \phi / d \xi=y$ and integrating it along the periodic orbit yield

$$
\begin{gathered}
\int_{\phi}^{\beta_{1}} \frac{\left(s-\phi_{s}\right) d s}{\left(s-\phi_{2}\right) \sqrt{\left(\beta_{1}-s\right)\left(s-\beta_{2}\right)\left(s-\beta_{3}\right)\left(s-\phi_{2}\right)}} \\
\quad=\sqrt{\frac{675 \Omega^{2}-9450 \Omega-84375}{24300 c^{2} \Omega}}|\xi| .
\end{gathered}
$$

Completing the above integral, we can get the parametric representation of smooth periodic wave solution as follows:

$$
\begin{gathered}
u(x, t)=\phi(x-c t)+\frac{4}{3} c, \\
\rho(x, t)=\left(\phi(x-c t)+\frac{1}{3} c\right)^{2} .
\end{gathered}
$$

$\phi$ is confirmed by

$$
\phi(\chi)=\frac{\beta_{1}\left(\beta_{2}-\phi_{2}\right)+\phi_{2}\left(\beta_{1}-\beta_{2}\right) \operatorname{sn}^{2}(\omega \chi, m)}{\left(\beta_{2}-\phi_{2}\right)+\left(\beta_{1}-\beta_{2}\right) \operatorname{sn}^{2}(\omega \chi, m)},
$$

$\xi(\chi)$

$$
\begin{aligned}
=\widetilde{\Omega} & {\left[\left(m^{2}-\alpha^{2}\right) \mathrm{F}(\arcsin (\operatorname{sn}(\omega \chi, m)), m)\right.} \\
& \left.+\alpha^{2} \mathrm{E}(\arcsin (\operatorname{sn}(\omega \chi, m)), m)-\frac{\omega m^{2}\left(\beta_{2}-\beta_{1}\right)}{\phi_{2}-\phi_{s}} \chi\right],
\end{aligned}
$$

where $\widetilde{\Omega}=\left(\left(\phi_{2}-\phi_{s}\right) / \omega m^{2}\left(\beta_{1}-\beta_{2}\right)\right)$ $\sqrt{24300 c^{2} \Omega /\left(675 \Omega^{2}-9450 \Omega-84375\right)}, \alpha^{2}=\left(\beta_{2}-\beta_{1}\right) /$ $\left(\beta_{2}-\phi_{2}\right), \omega=(1 / 2) \sqrt{\left(\beta_{1}-\beta_{3}\right)\left(\beta_{2}-\phi_{2}\right)}$, and $m=$ $\sqrt{\left(\beta_{1}-\beta_{2}\right)\left(\beta_{3}-\phi_{2}\right) /\left(\beta_{1}-\beta_{3}\right)\left(\beta_{2}-\phi_{2}\right)}$.

Remark 2. From Figures 3(f) and 3(n), we see that (2) has two solitary wave solutions when $(c, k) \in B_{6}$ and two solitary wave solutions when $(c, k) \in B_{14}$, but the exact parametric representations are very complex, so here we omit them. 


\section{Conclusion}

In this paper, using the bifurcation theory and the method of phase portraits analysis, we investigated the bifurcations of (2) (which is called a two-component Degasperis-Procesi equation) and obtained some exact parametric representations of peakon, solitary wave and smooth periodic wave solutions (see (23), (30), and (63), etc.). We can say that we obtained some new results of (2) in this paper.

\section{Acknowledgments}

The authors thank the referees very much for their perceptive comments and suggestions. This work is supported by the Natural Science Foundation of Yunnan Province, China (no. 2013FZ117) and the National Natural Science Foundation of China (no. 11161020).

\section{References}

[1] M. Chen, S. Q. Liu, and Y. Zhang, "A two-component generalization of the Camassa-Holm equation and its solutions," Letters in Mathematical Physics, vol. 75, no. 1, pp. 1-15, 2006.

[2] R. Camassa and D. D. Holm, "An integrable shallow water equation with peaked solitons," Physical Review Letters, vol. 71, no. 11, pp. 1661-1664, 1993.

[3] Y. Fu and C. Z. Qu, "Well posedness and blow-up solution for a new coupled Camassa-Holm equations with peakons," Journal of Mathematical Physics, vol. 50, no. 1, Article ID 012906, pp. 1-24, 2009.

[4] L. B. Jin and Z. G. Guo, "On a two-component DegasperisProcesi shallow water system," Nonlinear Analysis. Real World Applications, vol. 11, no. 5, pp. 4164-4173, 2010.

[5] M. Yuen, "Self-similar blowup solutions to the 2-component Degasperis-Procesi shallow water system," Communications in Nonlinear Science and Numerical Simulation, vol. 16, no. 9, pp. 3463-3469, 2011.

[6] A. Degasperis and M. Procesi, "Asymptotic integrability," in Symmetry and Perturbation Theory, pp. 23-37, World Scientific, Singapore, 1999.

[7] J. B. Li and H. H. Dai, On the Study of Singular Nonlinear Traveling Wave Equations: Dynamical System Approach, Science Press, Beijing, China, 2007.

[8] J. B. Li and Y. S. Li, "Bifurcations of travelling wave solutions for a two-component Camassa-Holm equation," Acta Mathematica Sinica, vol. 24, no. 8, pp. 1319-1330, 2008.

[9] B. He, "Bifurcations and exact bounded travelling wave solutions for a partial differential equation," Nonlinear Analysis: Real World Applications, vol. 11, no. 1, pp. 364-371, 2010.

[10] J. Li and Z. Liu, "Smooth and non-smooth traveling waves in a nonlinearly dispersive equation," Applied Mathematical Modelling, vol. 25, no. 1, pp. 41-56, 2000.

[11] P. F. Byrd and M. D. Friedman, Handbook of Elliptic Integrals for Engineers and Physicists, Springer, Berlin, Germany, 1971. 


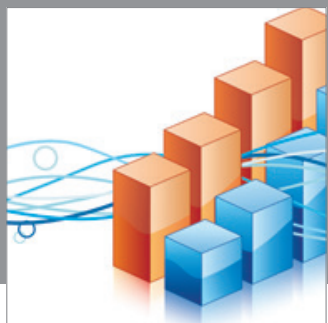

Advances in

Operations Research

mansans

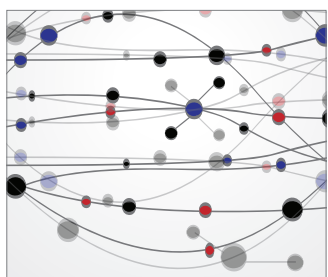

The Scientific World Journal
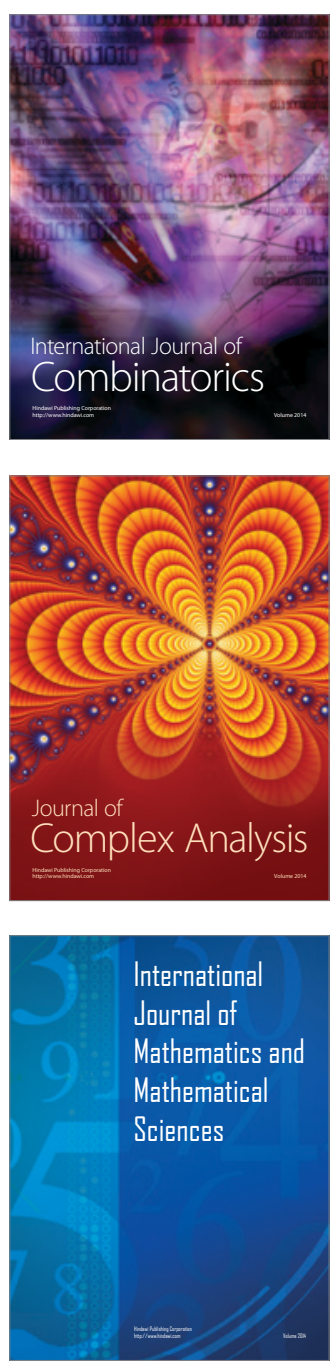
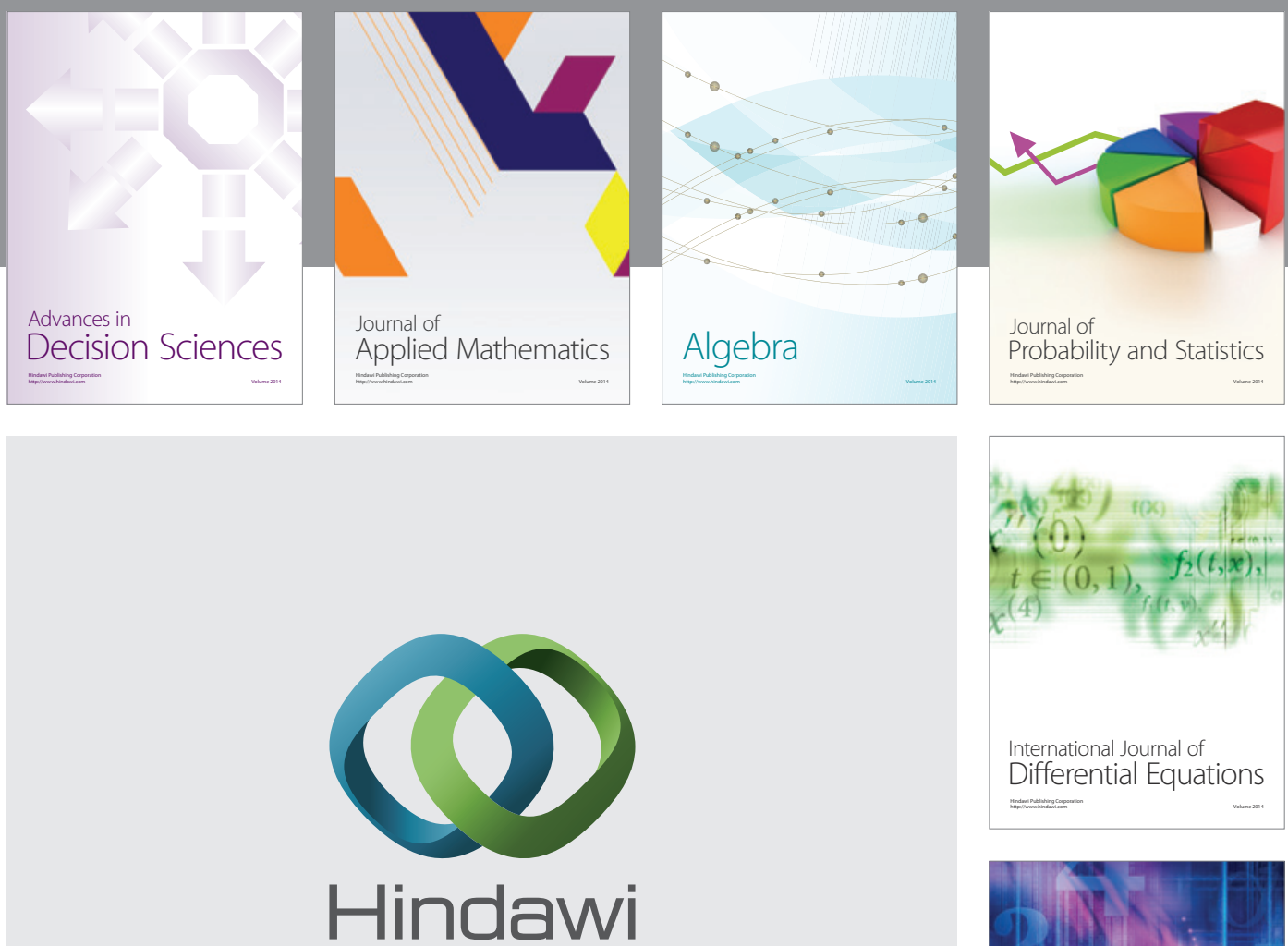

Submit your manuscripts at http://www.hindawi.com
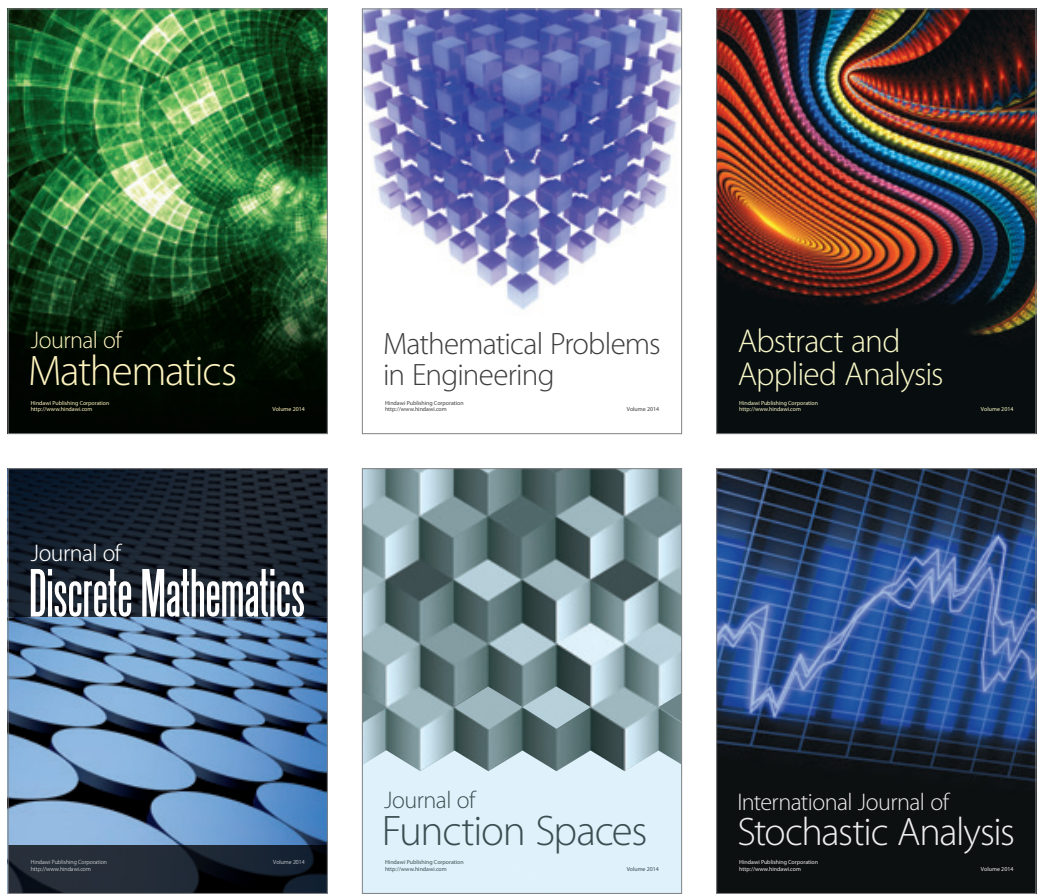

Journal of

Function Spaces

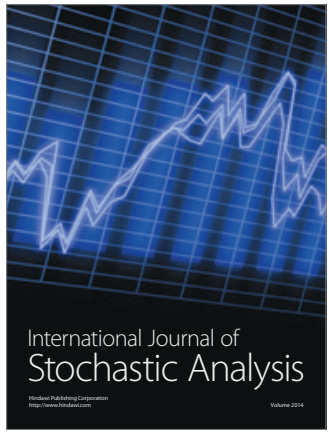

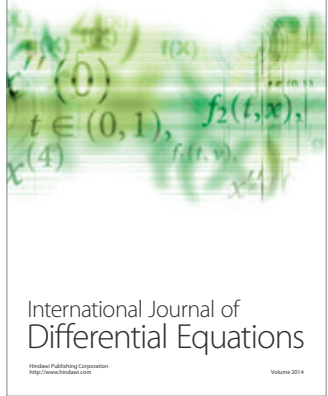
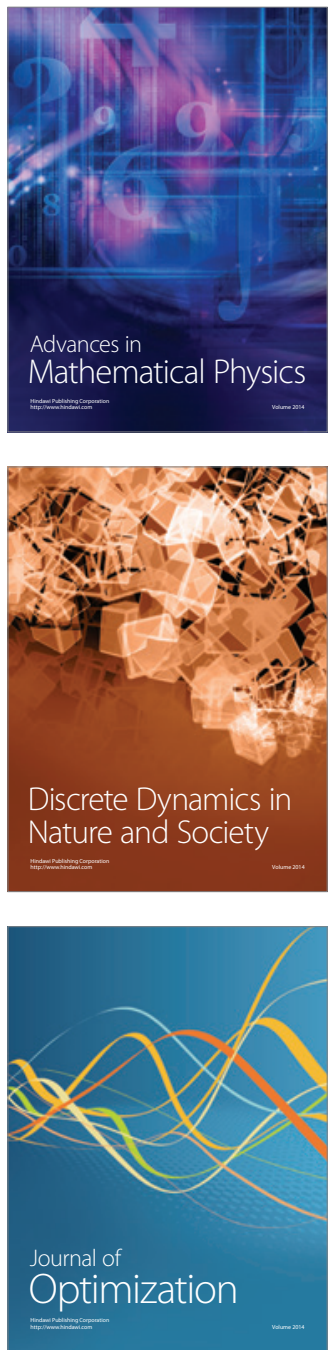